\title{
RESIDUES AND HOMOLOGY FOR PSEUDODIFFERENTIAL OPERATORS ON FOLIATIONS
}

\author{
M-T. BENAMEUR and V. NISTOR*
}

\begin{abstract}
We study the Hochschild homology groups of the algebra of complete symbols on a foliated manifold $(M, F)$. The first step is to relate these groups to the Poisson homology of $(M, F)$ and of other related foliated manifolds. We then establish several general properties of the Poisson homology groups of foliated manifolds. As an example, we completely determine these Hochschild homology groups for the algebra of complete symbols on the irrational slope foliation of a torus (under some diophantine approximation assumptions). We also use our calculations to determine all residue traces on algebras of pseudodifferential operators along the leaves of a foliation.
\end{abstract}

\section{Introduction}

This paper is a continuation of [2] and [3]. In those papers we have determined the Hochschild, cyclic, and periodic cyclic homology of certain algebras of complete symbols defined using groupoids. Our results were complete for periodic cyclic homology, these groups being given directly in terms of the cohomology of the cosphere bundle of the algebroid associated to our groupoid (this result is recalled in Theorem 5.2), for any differentiable groupoid.

The results for Hochschild homology groups (and hence also for cyclic homology groups) strongly depend, however, on the particular structure of the given groupoid. The previous two papers compute these groups for families of groupoids whose Lie algebroids are rationally isomorphic to the tangent bundle. This includes families of manifolds without boundary, families of $b$ pseudodifferential operators. We also treated in [3] the case of families of pseudodifferential operators on manifolds with corners.

In this paper, we study the Hochschild homology of algebras $\mathscr{A}(M, F)$ of complete symbols on a foliated manifold $(M, F)$. These algebras can also be defined using groupoids, although in this paper we choose to define them directly (see Section 5). We again obtain a convergent spectral sequence relating the Hochschild homology groups of $\mathscr{A}(M, F)$, denoted $\mathrm{HH}_{k}(\mathscr{A}(M, F))$,

\footnotetext{
* Nistor was partially supported by NSF Grant DMS-9971951 and "collaborative research" grant 9981251. Manuscripts available from http://www.math.psu.edu/nistor/.

Received April 4, 2002.
} 
$k=0,1, \ldots$, to the Poisson homology of $(M, F)$ and to the Poisson homology of various other foliations associated to $(M, F)$. This leads to a complete determination of the traces of $\mathscr{A}(M, F)$. These traces are usually called "residue traces." See [2], [3], [5], [11], [12], [14], [25], [26] for previous results of this kind. A motivation for the study of residue traces is our desire to understand an index theorem of Piazza for pseudodifferential operators on manifolds with boundary [21]. See also .

For simplicity, we have restricted ourselves here to foliated manifolds without boundary. In fact, the first three sections of this paper are devoted to the study of the Poissson homology of foliated manifolds and to their relation to longitudinal de Rham cohomology, as follows. We begin by reviewing some properties of de Rham cohomology groups for foliations in Section 1. Then, we discuss in Section 2 a Gysin long exact sequence for sphere fibrations of foliated manifolds following [22]. The homogeneous Poisson homology for conic foliated manifolds is defined and studied in Section 3. These homogeneous Poisson homology groups turn out to be isomorphic to certain de Rham cohomology groups, see Theorem 4.6. The corresponding result for non homogeneous homologies holds only for the longitudinal Poisson homology groups, see Definition 18 and [24].

In Section 5, we introduce the algebra $\mathscr{A}(M, F)$ of complete symbols on a foliated manifold $(M, F)$. We then compute in the last section, Section 6 , the $E^{2}$-term of a spectral sequence $\mathrm{EH}^{r}$ that we prove to converge to the Hochschild homology of longitudinal complete symbols. These computations show that if $p$ and $q$ are, respectively, the dimension and the codimension of the foliation $(M, F)$, then the groups

$$
\mathrm{HH}_{k}(\mathscr{A}(M, F))=0, \quad \text { if } \quad k>2 p+q .
$$

When the spectral sequence collapses at $E^{2}$, we get a complete computation. More precisely, in this case, the Hochschild homology groups of longitudinal complete symbols are given by (see Corollary 6.6):

$$
\mathrm{HH}_{k}(\mathscr{A}(M, F)) \simeq \bigoplus_{j=0}^{q} \mathrm{H}^{2 p+j-k, j}\left(\mathbf{S}^{*} F \times \mathrm{S}^{1}, F_{1}\right),
$$

where $S^{*} F$ is the longitudinal cosphere bundle and $F_{1}$ is the usual foliation on the total space of the bundle $\mathrm{S}^{*} F \times \mathrm{S}^{1}$ induced by $F$ (with same codimension).

For the lowest and the highest possibly non-trivial Hochschild homology groups, our results on the spectral sequence $\mathrm{EH}^{r}$, when combined with the Gysin exact sequence mentioned above, show that

$\mathrm{HH}_{0}(\mathscr{A}(M, F)) \simeq \mathrm{H}^{p, 0}(M, F)$ 


$$
\text { and } \mathrm{HH}_{2 p+q}(\mathscr{A}(M, F)) \simeq \mathrm{H}^{0, q}(M, F), \quad p \geq 2 .
$$

(See Theorem 6.3.) As a consequence, we obtain a bijective correspondence between residue traces and holonomy invariant transverse distributions, as expected.

In the last section, we determine the groups $\operatorname{HH}_{k}(\mathscr{A}(M, F))$ in the following particular case. Let $M=\left(\mathrm{S}^{1}\right)^{n}$ be foliated by the one parameter subgroups $\left(e^{2 \pi l \alpha_{1} t}, e^{2 \pi l \alpha_{2} t}, \ldots, e^{2 \pi l \alpha_{n} t}\right)$. We assume that the following Diophantine condition is satisfied: there exists $C>0$ and $N \in \mathrm{N}$ such that

$$
\left|m_{1} \alpha_{1}+m_{2} \alpha_{2}+\cdots+m_{n} \alpha_{n}\right|^{-1} \leq C\left(\left|m_{1}\right|+\left|m_{2}\right|+\cdots+\left|m_{n}\right|\right)^{N},
$$

for any $m_{1}, \ldots, m_{n} \in \mathbf{Z}$, not all zero. Then the Hochschild cohomology groups of this algebra are given by

$$
\mathrm{HH}^{l}(\mathscr{A}(M, F)) \cong \Lambda^{l} \mathrm{C}^{n+1} \otimes \mathrm{C}^{\{ \pm\}} .
$$

Note that even in this simple example, the assumption of Equation (1) is necessary for this determination to hold. In general, we need another formulation. This is in sharp contrast with the behavior of periodic cyclic homology groups.

We use several types of cohomology groups in this paper. The most important ones are introduced as follows:

- the longitudinal de Rham cohomology groups $\mathrm{H}^{r, s}(M, F), \mathrm{H}^{r, s}(M, F)_{l}$, and $\mathrm{H}_{c}^{r, s}(M, F)$ are introduced in Definition 1.2;

- the groups $\mathrm{H}^{k}(M, F)$ are introduced using Equation (6);

- the definition of the Poisson homology groups $\mathrm{H}_{k}^{\delta}(X)$ is recalled in Definition 3.2;

- the $l$ th homogeneous Poisson homology groups $\mathrm{H}_{k}^{\delta}(X)_{l}$ and $\mathrm{H}_{r, s}^{\delta_{F}}(X, \mathscr{F})_{l}$ are introduced in Definition 4.2; and, finally,

- the groups $\mathrm{H}_{k}^{\delta_{F}}(X)$ are introduced using Equation (18).

We assume $M$ to be compact for simplicity. Most of the following results and constructions work for $M$ non-compact by using cohomology with compact support. The proof is the same but notationally more complicated. In particular, the main computations of Hochschild homology, Theorems 6.2 and 6.3 remain true by considering compactly supported cohomology groups.

Acknowledgements. We thank Robert Lauter, Sergiu Moroianu, Jean Renault, Claude Roger, and Georges Skandalis for useful discussions. As we completed our manuscript, we received the preprint [13], which deals with some related questions. 


\section{1. de Rham cohomology for foliations}

Throughout this paper, we shall denote by $(M, F)$ a smooth manifold $M$ of dimension $n$ equipped with a smooth foliation $F$. So $F$ is, by definition, a smooth, integrable sub-bundle of the tangent bundle $T M$. The transverse bundle to the foliation $(M, F)$ is the quotient vector bundle $v=T M / F$. We denote by $p$ the dimension of $F$ and by $q$ the codimension of $F$. Thus $n=p+q$.

The sections of the longitudinal bundle $F$ will be called longitudinal vector fields. The sections of the exterior powers $\Lambda^{r} F^{*}$ of the dual vector bundle $F^{*}$ will be called longitudinal differential $r$-forms. The space of longitudinal differential $r$-forms will be denoted by $\Omega^{r, 0}(M, F)$ while $\Omega^{r}(M)$ will denote, as customary, the space of differential $r$-forms on the smooth manifold $M$. Every longitudinal vector field on $(M, F)$ is also a vector field on $M$ in the usual sense, therefore, any differential form on $M$ restricts to a longitudinal differential form on $(M, F)$. This defines surjections

$$
\Omega^{r}(M) \longrightarrow \Omega^{r, 0}(M, F) .
$$

More generally, a section of the bundle $\Lambda^{r} F^{*} \otimes \Lambda^{s} v^{*}$ will be called a differential form of bi-degree $(r, s)$, or $(r, s)$-differential form, for short. We denote the space of $(r, s)$-differential forms on $M$ by $\Omega^{r, s}(M, F)$.

Any choice of a supplementary sub-bundle $H$ to $F$ in $T M$ induces splittings

$$
\Theta_{H}: T^{*} M \cong F^{*} \oplus \nu^{*} \text { and } \Omega^{d}(M) \cong \bigoplus_{r+s=d} \Omega^{r, s}(M, F),
$$

obtained from the induced embeddings

$$
\Theta_{H}=\Theta_{H}^{r, s}: \Omega^{r, s}(M, F) \longrightarrow \Omega^{r+s}(M) .
$$

Note that $v^{*}$ identifies canonically with a sub-bundle of $T^{*} M$ (more precisely, with the annihilator of $F$ ). The splitting (2) endows $\Omega^{*}(M)$ with a bi-grading so that the de Rham differential decomposes as a sum of three bi-homogeneous components

$$
d=d_{F}+d_{\perp}+\partial
$$

where $d_{F}$ is the $(1,0)$-component, called the longitudinal differential, $d_{\perp}$ is the $(0,1)$-component and $\partial$ is an additional map that can be shown to have bi-degree $(-1,2)$ [23, page 35]. Moreover, $d_{F}$ does not depend on the choice of the complement $H$ to $F$ in $T M$, as we shall prove shortly.

In applications, spaces of compactly supported functions are also needed. Our constructions extend to this case with very few changes. For simplicity, we shall not consider this case separately. 
Let $Z \in \Gamma(v)$ be a section of the bundle $v$. We shall however denote by $Z_{H}$ the vector field in $\Gamma(H)$ that corresponds to $Z$ under the isomorphism $v \cong H$. Also, we shall denote by $\pi_{F}$ the projection $T M \rightarrow F$ with kernel $H$. Let $\theta$ be the smooth section of $F \otimes \Lambda^{2} v^{*}$ given for $Y, Z \in \Gamma(v)$ by

$$
\theta(Y, Z)=\pi_{F}\left(\left[Y_{H}, Z_{H}\right]\right)
$$

Recall that $\partial$ is the contraction by $\theta$, see [23] and also [8, page 267].

The equality $d^{2}=0$ is then equivalent to

$$
\begin{array}{ll}
d_{F}^{2}=0, \quad \partial^{2}=0, & d_{\perp}^{2}+\partial d_{F}+d_{F} \partial=0, \\
& d_{F} d_{\perp}+d_{\perp} d_{F}=0 \text { and } \partial d_{\perp}+d_{\perp} \partial=0 .
\end{array}
$$

Thus, for any $s \in\{0, \ldots, q\}$, we get the complex

(5) $0 \longrightarrow \Omega^{0, s}(M, F) \stackrel{d_{F}}{\longrightarrow} \Omega^{1, s}(M, F) \stackrel{d_{F}}{\longrightarrow} \cdots \stackrel{d_{F}}{\longrightarrow} \Omega^{p, s}(M, F) \longrightarrow 0$,

called the longitudinal de Rham complex. If $M$ is endowed with a free action of $\mathrm{R}_{+}^{*}$, we shall denote by

$$
\Omega^{r, s}(M, F)_{l} \subset \Omega^{r, s}(M, F)
$$

the subspace of forms that are homogeneous of degree $l$ with respect to the action of $\mathrm{R}_{+}^{*}$. We can assume that the action of $\mathrm{R}_{+}^{*}$ preserves the bundle $H$, and hence that the isomorphism $\Theta_{H}$ is invariant with respect to $\Theta_{H}$.

Lemma 1.1. The differential $d_{F}$ does not depend on the particular choice of $H$.

Proof. Let $H$ be the complement of $F$ used to define $d_{F}$. Denote by $\pi_{v}$ : $T M \rightarrow v \simeq H$ the quotient projection. For any $Y \in \Gamma(v)$, let $Y^{H} \in \Gamma(H)$ be the lifting of $Y$ to a vector field on $M$ such that $\pi_{v}\left(Y^{H}\right)=Y$.

Then the differential $d_{F}$ is explicitly given by

$$
\begin{aligned}
& d_{F} \omega\left(X_{1}, \ldots, X_{r+1} ; Y_{1}, \ldots, Y_{s}\right) \\
&= \sum_{j=1}^{r+1}(-1)^{s+j+1} X_{j} \omega\left(X_{1}, \ldots, \hat{X}_{j}, \ldots, X_{r+1} ; Y_{1}, \ldots, Y_{s}\right) \\
&+\sum_{1 \leq i<j \leq r+1} \omega\left(\left[X_{i}, X_{j}\right], X_{1}, \ldots, \hat{X}_{i}, \ldots, \hat{X}_{j}, \ldots, X_{r+1} ; Y_{1}, \ldots, Y_{s}\right) \\
&+\sum_{i=1}^{s} \sum_{j=1}^{r+1}(-1)^{s+j+i} \omega\left(X_{1}, \ldots, \hat{X}_{j}, \ldots, X_{r+1} ;\right. \\
&\left.\pi_{v}\left[Y_{i}^{H}, X_{j}\right], Y_{1}, \ldots, \hat{Y}_{i}, \ldots, Y_{s}\right) .
\end{aligned}
$$


where $\omega \in \Omega^{r, s}(M, F), X_{1}, \ldots, X_{r+1} \in \Gamma(F)$ and $Y_{1}, \ldots, Y_{s} \in \Gamma(v)$. Therefore, the only contribution of the splitting appears in the vector field $\pi_{\nu}\left[Y_{i}^{H}, X_{j}\right]$. But the projection $\pi_{\nu}\left[Y_{i}^{H}, X_{j}\right]$ actually does not depend on the particular choice of $H$, because $F$ is integrable. This completes the proof.

Definition 1.2. The $r^{\text {th }}$ cohomology space of the longitudinal de Rham complex (5) will be denoted by $\mathrm{H}^{r, s}(M, F)$. Similarly, we shall denote by $\mathrm{H}^{r, s}(M, F)_{l}$ the cohomology of the subcomplex of (5) consisting of $l$-homogeneous forms and by $\mathrm{H}_{c}^{r, s}(M, F)$ the cohomology of the subcomplex of (5) consisting of compactly supported forms.

We shall refer to all these groups as the longitudinal de Rham cohomology groups.

We shall also need the global longitudinal de Rham complex:

$$
0 \longrightarrow \Omega^{0}(M) \stackrel{d_{F}}{\longrightarrow} \Omega^{1}(M) \stackrel{d_{F}}{\longrightarrow} \cdots \stackrel{d_{F}}{\longrightarrow} \Omega^{n}(M) \longrightarrow 0,
$$

whose $k^{\text {th }}$ cohomology space is denoted $\mathrm{H}^{k}(M, F)$. So, using the splitting (2), we have:

$$
\mathrm{H}^{k}(M, F) \simeq \bigoplus_{r+s=k} \mathrm{H}^{r, s}(M, F) .
$$

The de Rham cohomology spaces of the smooth manifold $M$ will be denoted by $\mathrm{H}^{k}(M)$.

The homogeneity of $d_{F}, d_{\perp}$, and $\partial$ gives that

$$
d_{F}(\omega \wedge \eta)=d_{F} \omega \wedge \eta+(-1)^{r} \omega \wedge d_{F} \eta,
$$

where $\omega \in \Omega^{r, s}(M, F)$ and $\eta \in \Omega^{r^{\prime}, s^{\prime}}(M, F)$. Since

$$
\Theta_{H}\left(\Omega^{r, s}(M, F)\right) \wedge \Theta_{H}\left(\Omega^{r^{\prime}, s^{\prime}}(M, F)\right) \subset \Theta_{H}\left(\Omega^{r+r^{\prime}, s+s^{\prime}}(M, F)\right),
$$

we obtain a product

$$
\mathrm{H}^{r, s}(M, F) \otimes \mathrm{H}^{r^{\prime}, s^{\prime}}(M, F) \longrightarrow \mathrm{H}^{r+r^{\prime}, s+s^{\prime}}(M, F) .
$$

We shall also need functoriality properties for the groups $\mathrm{H}^{r, s}(M, F)$.

Proposition 1.3. Let $f:(M, F) \rightarrow\left(M_{1}, F_{1}\right)$ be a $\mathscr{C}^{\infty}$-map of foliated manifolds such that there exists complements $H$ and $H_{1}$ of $F$, respectively $F_{1}$, with $f_{*}(H) \subset H_{1}$. Then $f$ induces a map

$$
f^{*}: \mathrm{H}^{r, s}\left(M_{1}, F_{1}\right) \longrightarrow \mathrm{H}^{r, s}(M, F) .
$$


Proof. The assumption that $f:(M, F) \rightarrow\left(M_{1}, F_{1}\right)$ is a smooth map of foliated manifolds implies that $f$ induces a vector bundle morphism $f_{*}$ : $T M \rightarrow T M_{1}$ such that $f_{*}(F) \subset F_{1}$. The assumption that $f_{*}(H) \subset H_{1}$ then yields a map $\Gamma\left(H_{1}^{*}\right) \rightarrow \Gamma\left(H^{*}\right)$. Together with $\Gamma\left(F_{1}^{*}\right) \rightarrow \Gamma\left(F^{*}\right)$, these two maps give rise to the map

$$
f_{r, s}^{*}: \Omega^{r, s}\left(M_{1}, F_{1}\right) \longrightarrow \Omega^{r, s}(M, F) .
$$

Clearly $\Theta_{H}^{r, s} \circ f_{r, s}^{*}=f^{*} \circ \Theta_{H}^{r, s}$. Since $f^{*} \circ d=d \circ f^{*}$, by checking bi-degrees we see that

$$
d_{F} \circ f_{r, s}^{*}=f_{r+1, s}^{*} \circ d_{F_{1}} \text {. }
$$

This shows that the maps $f_{r, s}^{*}$ define a morphism of complexes, and hence they give rise to a map $f^{*}: \mathrm{H}^{r, s}\left(M_{1}, F_{1}\right) \rightarrow \mathrm{H}^{r, s}(M, F)$, as claimed.

Functoriality combine with the products (9) to define (external) products

$$
\mathrm{H}^{r, s}(M, F) \otimes \mathrm{H}^{r^{\prime}, s^{\prime}}\left(M_{1}, F_{1}\right) \rightarrow \mathrm{H}^{r+r^{\prime}, s+s^{\prime}}\left(M \times M_{1}, F \times F_{1}\right) .
$$

Note that $\mathrm{H}^{0, s}(M, F)$ coincides with the space $\Omega_{\mathrm{bas}}^{s}(M, F)$ of differential $s$-forms which are basic for the foliation, i.e. forms $\omega$ such that

$$
i_{Y} \omega=0 \text { and } L_{Y} \omega=0, \quad \forall Y \in \Gamma(F) .
$$

When restricted to differential forms of bi-degree $(0, *)$, the de Rham differential $d$ coincides with the sum $d_{F}+d_{\perp}$ so, using the equalities (4), we see that $d$ induces a well defined differential on $\mathrm{H}^{0, s}(M, F)$ that coincides with the differential induced by $d_{\perp}$. Thus the basic complex associated to $(M, F)$ is given by:

(11) $0 \longrightarrow \mathrm{H}^{0,0}(M, F) \stackrel{d=d_{\perp}}{\longrightarrow} \mathrm{H}^{0,1}(M, F) \stackrel{d_{\perp}}{\longrightarrow} \cdots \stackrel{d_{\perp}}{\longrightarrow} \mathrm{H}^{0, q}(M, F) \longrightarrow 0$.

The cohomology of this complex will be called, as customary, the basic de Rham cohomology of the foliated manifold $(M, F)$ and will be denoted by $\mathrm{H}_{\text {bas }}^{*}(M, F)$.

We shall use basic forms to study the behavior of the cohomology groups $\mathrm{H}^{r, s}$ with respect to some fibrations of foliated manifolds. To this end, we shall use a Leray spectral sequence with coefficients in the sheaf of germs of basic forms and the following well known result of I. Vaisman [24].

Proposition 1.4. The sequence of sheaves

$$
0 \longrightarrow \Omega_{\mathrm{bas}}^{h} \longrightarrow \Omega^{0, h} \stackrel{d_{F}}{\longrightarrow} \Omega^{1, h} \stackrel{d_{F}}{\longrightarrow} \cdots \stackrel{d_{F}}{\longrightarrow} \Omega^{p, h} \longrightarrow 0
$$

is a fine resolution of the sheaf $\Omega_{\mathrm{bas}}^{h}$ of basic $h$-forms. 
Therefore, the space $\mathrm{H}^{r, s}(M, F)$ can be identified with the $r^{t h}$ cohomology space of $M$ with coefficients in the sheaf $\Omega_{\mathrm{bas}}^{s}$.

COROLlary 1.5. For $0 \leq r \leq p$ and $0 \leq s \leq q$, we have

$$
\mathrm{H}^{r, s}(M, F) \cong \mathrm{H}^{r}\left(M, \Omega_{\mathrm{bas}}^{s}\right) .
$$

Similarly, $\mathrm{H}_{c}^{r, s}(M, F) \cong \mathrm{H}_{c}^{r}\left(M, \Omega_{\mathrm{bas}}^{s}\right)$.

\section{A Gysin exact sequence}

Let now $\pi: E \rightarrow M$ be a fiber bundle over the foliated manifold $(M, F)$. Let $F_{E}$ be the integrable sub-bundle of the tangent bundle $T E$ defined by

$$
F_{E}:=\operatorname{Ker}\left(p \circ \pi_{*}\right)
$$

where $p: T M \rightarrow T M / F$. To compute the bi-degree $(r, s)$ cohomologyspaces of the foliated manifold $\left(E, F_{E}\right)$, we can use a Gysin spectral sequence for the sheaf $\Omega_{\mathrm{bas}}^{s}$ over $E$.

The $E_{2}$ term of this spectral sequence is given by $E_{2}^{u, v}=\mathrm{H}^{u}\left(M, R^{v} \pi_{*}\left(\Omega_{\text {bas }}^{h}\right)\right)$, where the sheaf $R^{v} \pi_{*}\left(\Omega_{\text {bas }}^{h}\right)$ is defined by

$$
\left[R^{v} \pi_{*}\left(\Omega_{\mathrm{bas}}^{h}\right)\right](U)=\mathrm{H}^{v}\left(\pi^{-1}(U), \Omega_{\mathrm{bas}}^{h}\right) .
$$

Let us now recall the following result from [22], whose proof we include for the benefit of the reader.

Proposition 2.1 (Roger). Let $\pi: E \rightarrow M$ be any fibre bundle over $M$ with typical fiber a connected manifold $Y$. If $\mathscr{H}^{v}$ denotes the locally constant presheaf on $M$ defined by $\mathscr{H}^{v}(U)=\mathrm{H}^{v}\left(\pi^{-1}(U)\right)$ and $h$ is arbitrary, but fixed, then there exists a spectral sequence with

$$
E_{2}^{u, v} \cong \mathrm{H}^{u}\left(M, \Omega_{\text {bas }}^{h} \otimes \mathscr{H}^{v}\right)
$$

and convergent to $\mathrm{H}^{u+v, h}\left(E, F_{E}\right)$.

ProOF. Recall that a distinguished open covering of a foliated manifold $(M, F)$ is a covering of $M$ by open sets such that the induced foliation on each of these open sets is a product foliation with contractable fibers and contractable base. We can always find a distinguished open covering of the manifold $M$ that also trivializes the fibration $\pi: E \rightarrow M$. But with respect to any distinguished open set $U \cong W \times T, T$ transversal, such that $\pi^{-1}(U) \cong U \times Y \cong W \times T \times Y$, we have

$$
\Omega_{\mathrm{bas}}^{h}(W \times T \times Y) \simeq \Omega_{\mathrm{bas}}^{h}(W \times T) \otimes \mathrm{H}^{0}(Y) .
$$


This gives $\mathrm{H}^{u}\left(\pi^{-1}(U), \Omega_{\text {bas }}^{h}\right) \simeq \oplus_{u_{1}+u_{2}=u} \mathrm{H}^{u_{1}}\left(W \times T, \Omega_{\text {bas }}^{h}\right) \otimes \mathrm{H}^{u_{2}}(Y, \mathrm{R})$. On the other hand, we have: $\mathrm{H}^{u_{1}, h}(W \times T) \simeq 0$ if $u_{1}>0$, and

$$
\mathrm{H}^{0, h}(W \times T) \simeq \Omega_{\text {bas }}^{h}(W \times T) \simeq \Omega^{h}(T) .
$$

We thus obtain $\mathrm{H}^{u}\left(\pi^{-1}(U), \Omega_{\text {bas }}^{h}\right) \simeq \Omega_{\text {bas }}^{h}(W \times T) \otimes \mathrm{H}^{u}(Y, \mathrm{R})$. Our spectral sequence is the spectral sequence associated to the covering by the open sets $U$ above, and hence

$$
E_{2}^{u, v} \simeq \mathrm{H}^{u}\left(M, \Omega_{\text {bas }}^{h} \otimes \mathscr{H}^{v}\right)
$$

Now, let $E \stackrel{\pi}{\rightarrow} M$ be an oriented bundle with fiber of dimension $r$. Denote by $\pi^{*}$ the pull-back of differential forms and by $\pi_{*}$ integration along the fibres of $E \rightarrow M$. If $H$ is a splitting in $(M, F)$ as in (2), then $\pi^{*}(H)$ is a splitting for $\left(E, F_{E}\right)$. We fix these splittings in what follows.

LEMMA 2.2. (i) If $d_{F_{E}}$ is the longitudinal differential on the foliated manifold $\left(E, F_{E}\right)$, then

$$
d_{F_{E}} \circ \pi^{*}=\pi^{*} \circ d_{F} .
$$

(ii) Similarly, integration along the fibres $\pi_{*}$ satisfies

$$
d_{F} \circ \pi_{*}=\pi_{*} \circ d_{F_{E}} .
$$

Proof. (i) follows from Proposition 1.3.

(ii) In the same way, we deduce from the definition that $\pi_{*}$ is of bi-degree $(-r, 0)$, namely

$$
\pi_{*}: \Omega^{k, h}\left(E, F_{E}\right) \rightarrow \Omega^{k-r, h}(M, F) .
$$

Therefore, from the classical relation $\pi_{*} \circ d=d \circ \pi_{*}$ we deduce again by checking bi-degrees that $d_{F} \circ \pi_{*}=\pi_{*} \circ d_{F_{E}}$.

Remark 2.3. Assume that the fibers of $\pi: E \rightarrow M$ are diffeomorphic to the sphere $\mathrm{S}^{r}$ and that the fibration $E \rightarrow M$ is oriented. The Euler class $e \in \mathrm{H}^{r+1}(M)$ is then defined (see [15] for details). Moreover, it can be represented by an element of $\mathrm{H}^{r+1,0}(M, F)$ (this actually follows from the proof of Theorem 2.4). Therefore, we have for any $\alpha \in \Omega^{k, h}(M, F)$ that

$$
d_{F}(\alpha \wedge e)=d_{F}(\alpha) \wedge e .
$$

In the sequel, in order to make our results more explicit, we shall need a Gysin exact sequence for the $(k, h)$-cohomology groups. More precisely, we have 
Theorem 2.4. Assume that $\pi: E \rightarrow M$ is an oriented sphere bundle with fiber $\mathrm{S}^{r}$ and denote by $e \in \mathrm{H}^{r+1,0}(M)$ the Euler class of this bundle, then, for any $h$ in the range $0 \leq h \leq q$, we have the following Gysin exact sequence

$$
\begin{aligned}
\cdots \stackrel{\pi^{*}}{\longrightarrow} & \mathrm{H}^{k, h}\left(E, F_{E}\right) \stackrel{\pi_{*}}{\longrightarrow} \mathrm{H}^{k-r, h}(M, F) \stackrel{\wedge e}{\longrightarrow} \mathrm{H}^{k+1, h}(M, F) \\
& \stackrel{\pi^{*}}{\longrightarrow} \mathrm{H}^{k+1, h}\left(E, F_{E}\right) \stackrel{\pi_{*}}{\longrightarrow} \cdots
\end{aligned}
$$

Proof. Because $E$ is an oriented bundle, the presheaf $\mathscr{H}^{v}$ has no monodromy (that is, it is constant). Thus, we obtain

$$
E_{2}^{u, v} \cong \mathrm{H}^{u, h}(M) \otimes \mathrm{H}^{v}\left(\boldsymbol{S}^{r}\right) .
$$

Now since $\mathrm{H}^{v}\left(\boldsymbol{S}^{r}\right)=0$ if $v \neq 0$ and $v \neq r$, we get inclusions $E_{\infty}^{k-r, r} \hookrightarrow E_{2}^{k-r, r}$, $\forall k \geq 0$. Therefore the following sequence is exact

$$
0 \longrightarrow E_{\infty}^{k-r, r} \hookrightarrow E_{2}^{k-r, r} \stackrel{d_{r+1}}{\longrightarrow} E_{2}^{k+1,0} \longrightarrow E_{\infty}^{k+1,0} \longrightarrow 0 .
$$

On the other hand we have an exact sequence

$$
0 \longrightarrow E_{\infty}^{k, 0} \longrightarrow \mathrm{H}^{k, h}\left(E, F_{E}\right) \longrightarrow E_{\infty}^{k-r, r} \longrightarrow 0 .
$$

But $E_{2}^{k-r, r} \simeq \mathrm{H}^{k-r, h}(M, F)$ and $E_{2}^{k+1,0} \simeq \mathrm{H}^{k+1, h}(M, F)$. As in the case of the classical Gysin sequence, the above two short exact sequences yield a long exact sequence,

$$
\begin{aligned}
\cdots \longrightarrow \mathrm{H}^{k, h}\left(E, F_{E}\right) \longrightarrow & \mathrm{H}^{k-r, h}(M, F) \longrightarrow \mathrm{H}^{k+1, h}(M, F) \\
& \longrightarrow \mathrm{H}^{k+1, h}\left(E, F_{E}\right) \longrightarrow \mathrm{H}^{k+1-r, h}(M, F) \longrightarrow \cdots
\end{aligned}
$$

To end the proof, we must identify the maps involved in this exact sequence. But this is again similar to the computation for the classical Gysin sequence.

From this theorem we obtain the following corollaries.

Corollary 2.5. We use the notation of Theorem 2.4.

(i) The map $\pi^{*}: \mathrm{H}^{k, h}(M, F) \longrightarrow \mathrm{H}^{k, h}\left(E, F_{E}\right)$ is an isomorphism for any $r \geq 1$ and $0 \leq k \leq r-1$.

(ii) The map $\pi_{*}: \mathrm{H}^{k, h}\left(E, F_{E}\right) \longrightarrow \mathrm{H}^{k-r, h}(M, F)$ is an isomorphism for any $k \geq p+1, p=\operatorname{dim}(F)$.

PROOF. This is a corollary of the longitudinal Gysin exact sequence proved in Theorem 2.4. More precisely, for $k \leq r-1$ we have $\mathrm{H}^{k-r, h}(M, F)=0$. 
Therefore, we get:

$$
\begin{aligned}
& \cdots \stackrel{\pi_{*}}{\longrightarrow} \mathrm{H}^{k-r-1, h}(M, F)=0 \stackrel{\wedge e}{\longrightarrow} \mathrm{H}^{k, h}(M, F) \stackrel{\pi^{*}}{\longrightarrow} \mathrm{H}^{k, h}\left(E, F_{E}\right) \\
& \stackrel{\pi_{*}}{\longrightarrow} \mathrm{H}^{k-r, h}(M, F)=0 \stackrel{\wedge e}{\longrightarrow} \cdots
\end{aligned}
$$

In the same way, if $k \geq p+1$, then $\mathrm{H}^{k, h}(M, F)=0$, therefore we get:

$$
\begin{aligned}
\cdots \stackrel{\wedge e}{\longrightarrow} \mathrm{H}^{k, h}(M, F)=0 \stackrel{\pi^{*}}{\longrightarrow} \mathrm{H}^{k, h}\left(E, F_{E}\right) \stackrel{\stackrel{\pi_{*}}{\longrightarrow}}{\longrightarrow} \mathrm{H}^{k-r, h}(M, F) \\
\stackrel{\wedge e}{\longrightarrow} \mathrm{H}^{k+1, h}(M, F)=0 \stackrel{\pi^{*}}{\longrightarrow} \cdots
\end{aligned}
$$

In particular, for the product $E=M \times \mathrm{S}^{r}$, we get the following isomorphism that will be used later on.

Corollary 2.6. If $E=M \times \mathrm{S}^{r}$, then

$$
\mathrm{H}^{k, h}\left(E, F_{E}\right) \simeq \mathrm{H}^{k, h}(M, F) \oplus \mathrm{H}^{k-r, h}(M, F),
$$

naturally.

Proof. The Euler class $e$ vanishes because $E$ is a product, and hence the Gysin long exact sequence of Theorem 2.4 decomposes as a direct sum of short exact sequences

$$
0 \longrightarrow \mathrm{H}^{k, h}(M, F) \longrightarrow \mathrm{H}^{k, h}\left(E, F_{E}\right) \longrightarrow \mathrm{H}^{k-r, h}(M, F) \longrightarrow 0 .
$$

To complete the proof, it is enough to prove that the above sequence splits naturally. To this end, let $\omega_{r}$ be the generator of $\mathrm{H}^{r}\left(\mathbf{S}^{r}\right)$. We can pull this class to a cohomology class in $\mathrm{H}^{r, 0}\left(E, F_{E}\right)$, denoted $\eta_{r}$. Then the product with $\eta_{r}$ defines the desired natural splitting $\mathrm{H}^{k-r, h}(M, F) \rightarrow \mathrm{H}^{k, h}\left(E, F_{E}\right)$.

For $\alpha>0$, the vector bundle $|\Lambda|^{\alpha}(M)$ of $\alpha$-densities over $M$ is, by definition, the line bundle whose fiber at a point $x$ is the 1-dimensional complex vector space of maps $\rho: \Lambda^{n}\left(T_{x} M\right) \rightarrow C$ that satisfy

$$
\forall \lambda \in \mathrm{R}, \forall v \in \Lambda^{n}\left(T_{x} M\right), v \neq 0, \quad \rho(\lambda v)=|\lambda|^{\alpha} \rho(v) .
$$

This bundle admits nowhere vanishing sections and is, in fact, trivializable, but not in a canonical way, in general. Denote by $C_{M}$ the complex orientation bundle of $T M$, then we have

$$
|\Lambda|^{1}(M) \cong \Lambda^{n} T^{*} M \otimes \mathrm{C}_{M} .
$$

Let now $E$ be a smooth (real) vector bundle over $M$. The space of compactly supported smooth sections of $E$ is then naturally endowed with a structure of 
a locally convex space. The space of generalized sections of the vector bundle $E$ is by definition the dual space of the space of compactly supported smooth sections of the vector bundle $E^{*} \otimes\left|\Lambda^{1}\right|(M)$, where $E^{*}$ is the dual vector bundle of $E$. Hence a distribution on $M$ can also be viewed as a generalized 1-density. Some functorial properties of generalized sections are studied in [10]. In particular, the pull-back of generalized sections is well defined for fibrations (by integration along the fibers).

A $k$-current on $M$ is a generalized section of the bundle $\Lambda^{n-k}\left(T^{*} M\right) \otimes C_{M}$. So, a $k$-current on $M$ is, by definition, a continuous linear form on the space

$$
C_{c}^{\infty}\left(M, \Lambda^{n-k}(T M) \otimes C_{M}^{*} \otimes\left|\Lambda^{1}\right|(T M)\right) .
$$

But since,

$\left|\Lambda^{1}\right|(T M) \cong \Lambda^{n}\left(T^{*} M\right) \otimes C_{M} \quad$ and $\quad \Lambda^{n-k}(T M) \otimes \Lambda^{n}\left(T^{*} M\right) \cong \Lambda^{k}\left(T^{*} M\right)$, we get

$$
\Lambda^{n-k}(T M) \otimes C_{M}^{*} \otimes\left|\Lambda^{1}\right|(T M) \cong \Lambda^{k}\left(T^{*} M\right) \otimes C_{M}^{*} \otimes C_{M} .
$$

This shows that any $k$-current $\phi$ defines a linear map

$$
\phi: \mathscr{C}_{c}^{\infty}\left(M, \Lambda^{k} T^{*} M\right)=: \Omega^{k}(M) \rightarrow \mathrm{C} .
$$

Denote, as before, by $v$ the transverse vector bundle $v=T M / F$. We define a $(k, h)$-current as a generalized section of the bundle

$$
\Lambda^{p-k}\left(F^{*}\right) \otimes \Lambda^{q-h}\left(v^{*}\right) \otimes C_{M} .
$$

We shall denote the space of $(k, h)$-currents by $A_{k, h}(M, F)$.

Lemma 2.7. By choosing a transverse distribution $H$, we can view any $(k, h)$-current as a continuous linear form on the space of compactly supported differential $(k, h)$-forms.

Proof. A $(k, h)$-current on $M$ is by definition a continuous linear form on the space

$$
C_{c}^{\infty}\left(M, \Lambda^{p-k}(F) \otimes \Lambda^{q-h}(v) \otimes C_{M}^{*} \otimes\left|\Lambda^{1}\right|(T M)\right) .
$$

The choice of $H$ fixes an isomorphism $T M \cong F \oplus v$ so that

$$
\left|\Lambda^{1}\right|(T M) \cong \Lambda^{n}\left(T^{*} M\right) \otimes C_{M} \cong \Lambda^{p}\left(F^{*}\right) \otimes \Lambda^{q}\left(v^{*}\right) \otimes C_{M} .
$$

Using

$$
\Lambda^{p-k}(F) \otimes \Lambda^{p}\left(F^{*}\right) \cong \Lambda^{k}\left(F^{*}\right) \quad \text { and } \quad \Lambda^{q-h}(v) \otimes \Lambda^{q}\left(v^{*}\right) \cong \Lambda^{h}\left(v^{*}\right),
$$


we obtain that

$$
\Lambda^{p-k}(F) \otimes \Lambda^{q-h}(v) \otimes C_{M}^{*} \otimes\left|\Lambda^{1}\right|(T M) \cong \Lambda^{k}\left(F^{*}\right) \otimes \Lambda^{h}\left(v^{*}\right) \otimes C_{M}^{*} \otimes C_{M}
$$

To finish the proof, we use that the bundle $\mathrm{C}_{M}^{*} \otimes \mathrm{C}_{M}$ is canonically isomorphic to the trivial line bundle.

The above lemma shows, in particular, that orientation-twisted ( $p-k, q-$ $h$ )-differential forms define a pairing with $(k, h)$-differential forms. This is, of course, nothing but the Poincaré map.

For a fixed transverse distribution $H$, we define a longitudinal differential on the space of $(k, h)$-currents, still denoted $d_{F}$, satisfying $d_{F}^{2}=0$, which again does not depend on the particular choice of $H$. This differential is dual to the one defined above on smooth differential forms and we get in this way longitudinal complexes $\left(A_{*, h}(M, F), d_{F}\right)_{0 \leq h \leq q}$ of currents:

$$
0 \longrightarrow A_{p, h} \longrightarrow A_{p-1, h} \longrightarrow \cdots \longrightarrow A_{0, h} \longrightarrow 0 \text {. }
$$

The cohomology of this complex will be denoted $\mathrm{H}_{*, h}(M, F)$. So we have a duality map $\mathrm{H}_{k, h}(M, F) \rightarrow\left[\mathrm{H}^{k, h}(M, F)\right]^{\prime}$, where $\mathrm{H}^{k, h}(M, F)$ is endowed with the quotient topology.

We include now some remarks that are useful for the reader interested in relating the above constructions to transverse measures on foliations.

Definition 2.8. Let $(M, F)$ be a smooth foliated manifold of dimension $p$ and codimension $q=n-p$, as before, and let $v=T M / F$ be the transverse vector bundle.

(i) A transverse current $C$ on $(M, F)$ is a current of bi-degree $(p, k)$ for $0 \leq k \leq q$, i.e. a generalized section of the bundle $\Lambda^{q-k}\left(v^{*}\right) \otimes \mathrm{C}_{M}$.

(ii) An invariant current on $(M, F)$ is a current $C$ on $M$ such that $d_{F}(C)=0$.

(iii) A current which is transverse and invariant is also called a basic current.

Note that a basic current of type $(p, 0)$ is automatically closed in $M$. Note also that invariant currents are are also sometimes called holonomy invariant currents, see [1]. The simplest example of a transverse current is the RuelleSullivan current associated with any holonomy invariant transverse measure on $(M, F)$. Recall that a transverse measure on $(M, F)$ is a $\sigma$-finite measure on the disjoint union of submanifolds of $M$ which are everywhere transverse to the foliation. A transverse measure will be called an invariant transverse measure if it is invariant under the action of the holonomy pseudogroup [20]. Given an invariant transverse measure $\mu$, we canonically associate to $\mu$ an element $C_{\mu}$ of $C^{-\infty}\left(M, \Lambda^{q} \otimes \mathrm{C}_{v}\right)=C^{-\infty}\left(M,\left|\Lambda^{1}\right|\left(v^{*}\right)\right)$ by using partitions 
of unity. Therefore, if the foliation is oriented, we have $\mathrm{C}_{M}=\mathrm{C}_{v}$ and $\mu$ gives rise to a basic current of dimension $q$ that is closed in $M$.

\section{Canonical homology for foliations}

We begin this section by recalling the Koszul-Brylinski complex [4] of a foliated Poisson manifold and also some of its properties that will be needed in the sequel. Let $(M, F)$ be a smooth foliation with $\operatorname{dim}(M)=n, \operatorname{dim}(F)=p$, and $\operatorname{codim}(F)=q$, as before. We are interested in the manifold $X=F^{*} \backslash M$, the dual of $F$ with the zero section (identified with $M$ ) removed. Then $X$ acquires a natural foliation $\mathscr{F}$ of dimension $2 p$ and codimension $q$. Moreover, $X$ admits an additional structure, that of a "foliated Poisson manifold," which we proceed now to define. In the whole section $(X, \mathscr{F})$ will then be a foliated manifold whose leaves have dimension $2 p$ and codimension $q$. We shall insist that $X=F^{*}$ or some submanifold of $F^{*}$ when necessary.

Definition 3.1. A foliated Poisson structure on $(X, \mathscr{F})$ is a (foliated) 2tensor $G \in \Gamma\left(\Lambda^{2} \mathscr{F}\right) \subset \Gamma\left(\Lambda^{2} T M\right)$ over $X$ such that the Schouten-Nijenhuis bracket $[G, G]_{S N}$ is trivial, see [24].

A foliated Poisson structure $G$ gives rise to a bilinear form $\{\cdot, \cdot\}$ on the algebra $\mathscr{C}^{\infty}(X)$ of smooth maps on $X$, called the Poisson bracket and defined by the formula

$$
\{f, g\}=i_{G}(d f \wedge d g)
$$

where $d$ is the de Rham differential on the smooth manifold $X$ and $i_{G}$ is the interior product by the 2-tensor $G$. The condition $[G, G]_{S N}=0$ then corresponds to the assumption that $\{\cdot, \cdot\}$ defines a Lie algebra structure on $\mathscr{C}^{\infty}(X)$. Since for any $f \in \mathscr{C}^{\infty}(X)$, the map $g \mapsto\{f, g\}$ is a derivation of the commutative ring underlying $\mathscr{C}^{\infty}(X)$, a foliated Poisson structure on $X$ endows it with the structure of a Poisson manifold. Note that the Hamiltonian vector fields associated with a foliated Poisson structure are tangent to the leaves of the foliation $(X, \mathscr{F})$. The symplectic leaves of a foliated Poisson manifold $(X, \mathscr{F}, G)$ are contained in the foliation $\mathscr{F}$. When this foliation coincides with the original foliation $\mathscr{F}$, we say that the Poisson foliated manifold $(X, \mathscr{F}, G)$ is a longitudinally symplectic foliated manifold.

A Poisson manifold is a foliated Poisson manifold for any regular foliation that contains the (singular in general) symplectic foliation. Foliated Poisson manifolds are especially interesting when the symplectic foliation of a given Poisson manifold can be embedded in a regular foliation of small dimension. A regular Poisson manifold $M$, i.e. with a regular symplectic foliation, is a 
foliated Poisson manifold for the symplectic foliation itself, but also for any other foliation that contains the symplectic one.

An important example for our purposes is that of the cotangent bundle of any smooth foliation. More precisely, let $(M, F)$ be a smooth foliated manifold and denote by $\pi_{v}: T M \rightarrow T M / F=: v$ the quotient map. Let $X:=F^{*}$ be the total space of the longitudinal cotangent bundle to $(M, F)$ and denote by $\pi: X \rightarrow M$ the canonical projection. The kernel of the composite map $\pi_{v} \circ \pi_{*}: T X \rightarrow T M / F$ is then an integrable sub-bundle $\mathscr{F}$ of the tangent bundle $T X$ to $X$. The leaves of the resulting foliation $\mathscr{F}$ on $X$ are exactly the restrictions of the bundle $F^{*}$ to the leaves of $(M, F)$ and so are symplectic manifolds. By putting together the resulting symplectic 2-tensors, we obtain a longitudinally symplectic foliated manifold $(X, \mathscr{F})$.

Let now $(X, \mathscr{F}, G)$ be a general foliated Poisson manifold. The Poisson differential $\delta$, is defined as for any Poisson manifold by the formula [4]

$$
\delta:=i_{G} \circ d-d \circ i_{G}: \Omega^{k}(X) \longrightarrow \Omega^{k-1}(X) .
$$

We now recall the definition of Poisson homology of the Poisson foliation $(X, \mathscr{F})$.

Definition 3.2. We denote by $\mathrm{H}_{k}^{\delta}(X)$ the Poisson homology of $X$, defined by

$$
\mathrm{H}_{k}^{\delta}(X):=\frac{\operatorname{Ker}\left(\delta: \Omega^{k}(X) \rightarrow \Omega^{k-1}(X)\right)}{\delta\left(\Omega^{k+1}(X)\right)} .
$$

Assume that we have fixed a splitting $\Theta_{H}$ as in (2) for the foliated manifold $(X, \mathscr{F})$. This, in turn, fixes isomorphisms $\Omega^{k}(X) \simeq \bigoplus_{r+s=k} \Omega^{r, s}(X, \mathscr{F})$.

Lemma 3.3. [24] Let $(X, \mathscr{F}, G)$ be a foliated Poisson manifold, then the Koszul-Brylinski operator $\delta$, has a canonical decomposition into two bi-homogeneous operators

$$
\delta=\delta_{\mathscr{F}}+\delta_{-2,1},
$$

where $\delta_{\mathscr{F}}=\left[i_{G}, d_{\mathscr{F}}\right]$ is a component of bi-degree $(-1,0)$ with respect to the splitting, called the longitudinal Poisson differential, and $\delta_{-2,1}$ is an extra term with bi-degree $(-2,1)$ with respect to the bi-grading. Furthermore, if $d=d_{\mathscr{F}}+d_{\perp}+\partial$, as in Equation (3) with $(X, \mathscr{F})$ in place of $(M, F)$, we have

$$
\delta_{-2,1}=\left[i_{G}, d_{\perp}\right], \quad \delta_{\mathscr{F}}^{2}=0, \quad \delta_{-2,1}^{2}=0, \quad \text { and } \quad \delta_{\mathscr{F}} \delta_{-2,1}+\delta_{-2,1} \delta_{\mathscr{F}}=0 .
$$

Proof. Let $H$ be a supplementary sub-bundle to $\mathscr{F}$ in $T X$ and $d=d_{\mathscr{F}}+$ $d_{\perp}+\partial$ the corresponding decomposition of the de Rham operator $d$ as recalled in Section 1. Let us show that $\left[i_{G}, \partial\right]=0$. Let $X \in \Gamma(\mathscr{F})$ be a longitudinal 
vector field. Then, for any $X_{1}, \ldots, X_{k-1} \in \Gamma(\mathscr{F})$, for any $Y_{1}, \ldots, Y_{h+2} \in$ $\Gamma(H) \simeq \Gamma(v)$, and for any $\omega \in \Omega^{k+1, h}(X, \mathscr{F})$, we have:

$$
\begin{aligned}
& \partial\left(i_{X} \omega\right)\left(X_{1}, \ldots, X_{k-1} ; Y_{1}, \ldots, Y_{h+2}\right)=\sum_{1 \leq j<i \leq h+2} \\
& \quad(-1)^{i+j+h} \omega\left(X, \pi_{\mathscr{F}}\left[Y_{j}, Y_{i}\right], X_{1}, \ldots, X_{k-1} ; Y_{1}, \ldots, \hat{Y}^{j}, \ldots, \hat{Y}^{i}, \ldots, Y_{h+2}\right) .
\end{aligned}
$$

On the other hand:

$$
\begin{aligned}
& i_{X}(\partial \omega)\left(X_{1}, \ldots, X_{k-1} ; Y_{1}, \ldots, Y_{h+2}\right)=\sum_{1 \leq j<i \leq h+2} \\
& (-1)^{i+j+h} \omega\left(\pi_{\mathscr{F}}\left[Y_{j}, Y_{i}\right], X, X_{1}, \ldots, X_{k-1} ; Y_{1}, \ldots, \hat{Y}^{j}, \ldots, \hat{Y}^{i}, \ldots, Y_{h+2}\right) .
\end{aligned}
$$

Thus we deduce that $i_{X} \circ \partial+\partial \circ i_{X}=0$ and hence

$$
\left[i_{X \wedge Y}, \partial\right]=i_{Y}\left(i_{X} \partial+\partial i_{X}\right)-\left(i_{Y} \partial+\partial i_{Y}\right) i_{X}=0,
$$

for any $(X, Y) \in \Gamma(\mathscr{F})$. Therefore $\left[i_{A}, \partial\right]=0$, for all $A \in \Gamma\left(\Lambda^{2} \mathscr{F}\right)$. We finish the proof by setting $\delta_{-2,1}=\left[i_{G}, d_{\perp}\right]$. Finally the identity $\delta^{2}=0$ [4] gives the claimed equalities by direct inspection of the bi-degrees. See also [24, Proposition 4.13].

REMARK 3.4. The contraction by $G$ has bi-degree $(-2,0)$ and satisfies the relation

$i_{G}\left(\omega_{1} \wedge \omega_{2}\right)=i_{G}\left(\omega_{1}\right) \wedge \omega_{2}, \quad \forall \omega_{1} \in \Gamma\left(\Lambda^{*} T^{*} X\right) \quad$ and $\quad \forall \omega_{2} \in \Gamma\left(\Lambda^{*} H^{*}\right)$.

Assume for the rest of this section that $(X, \mathscr{F})$ is a longitudinally symplectic foliation with $\operatorname{dim}(\mathscr{F})=2 p$ and $\operatorname{codim}(\mathscr{F})=q$.

If $U$ is a distinguished chart for the foliation $(X, \mathscr{F})$, then $\delta_{\mathscr{F}}$ restricts to $U$ and induces a well defined differential on the sheaf of germs of smooth longitudinal differential forms. The action of $\delta \mathscr{F}$ on typical longitudinal forms is similar to the classical one. More precisely:

Proposition 3.5. Let $(X, \mathscr{F}, G)$ be a general foliated Poisson manifold. Then the action of $\delta_{\mathscr{F}}$ on typical longitudinal forms is given by

$$
\begin{aligned}
\delta_{\mathscr{F}}\left(f_{0} d_{\mathscr{F}} f_{1} \ldots d_{\mathscr{F}} f_{k}\right)=\sum_{1 \leq j \leq k}(-1)^{j+1}\left\{f_{0}, f_{j}\right\} d_{\mathscr{F}} f_{1} \ldots \widehat{d_{\mathscr{F}}} f_{j} \ldots d_{\mathscr{F}} f_{k} \\
+\sum_{1 \leq i<j \leq k}(-1)^{i+j} f_{0} d \mathscr{F}\left\{f_{i}, f_{j}\right\} d_{\mathscr{F}} f_{1} \ldots \widehat{d_{\mathscr{F}} f_{i}} \ldots \widehat{d_{\mathscr{F}}} f_{j} \ldots d_{\mathscr{F}} f_{k},
\end{aligned}
$$

for all $f_{0}, \ldots, f_{k} \in \mathscr{C}^{\infty}(X)$. 
Proof. The computations carried out in [4, page 96] imply our proposition. Recall that we have

$$
\begin{aligned}
\delta\left(f_{0} d f_{1} d f_{2} \ldots d f_{k}\right)= & \sum_{1 \leq j \leq k}(-1)^{j+1}\left\{f_{0}, f_{j}\right\} d f_{1} \ldots \widehat{d f}_{j} \ldots d f_{k} \\
& +\sum_{1 \leq i<j \leq k}(-1)^{i+j} f_{0} d\left\{f_{i}, f_{j}\right\} d f_{1} \ldots \widehat{d f}_{i} \ldots \widehat{d f}_{j} \ldots d f_{k},
\end{aligned}
$$

for all $f_{0}, f_{1}, \ldots, f_{k} \in \mathscr{C}^{\infty}(X)$. Hence taking the $(k-1,0)$ component of each side of the above equality gives exactly the allowed formula.

Let us also mention, for completeness, the following result.

Proposition 3.6.

(i) If $\omega \in \Gamma\left(\Lambda^{k} \mathscr{F}^{*}\right)$ and $\omega^{\prime} \in \Gamma\left(\Lambda^{k^{\prime}} H^{*}\right)$, then we have

$$
\delta_{-2,1}\left(\omega \wedge \omega^{\prime}\right)=\delta_{-2,1}(\omega) \wedge \omega^{\prime}
$$

(ii) For any $X_{1}, \ldots, X_{r} \in \Gamma(\mathscr{F})$, for any $Z \in \Gamma(H)$, and for any $\omega \in$ $\Omega^{r+2,0}(X, \mathscr{F})$,

$$
\delta_{-2,1} \omega\left(X_{1}, \ldots, X_{r}, Z\right)=i_{[G, Z]_{S N}} \omega\left(X_{1}, \ldots, X_{r}\right) .
$$

Proof. (i) For any $\omega \in \Gamma\left(\Lambda^{k} \mathscr{F}^{*}\right)$ and any $\omega^{\prime} \in \Gamma\left(\Lambda^{k^{\prime}} H^{*}\right)$, we have

$$
d_{\perp}\left(\omega \wedge \omega^{\prime}\right)=d_{\perp}(\omega) \wedge \omega^{\prime}+(-1)^{k} \omega \wedge d_{\perp}\left(\omega^{\prime}\right) .
$$

Therefore

$$
\left[i_{G}, d_{\perp}\right]\left(\omega \wedge \omega^{\prime}\right)=i_{G}\left(d_{\perp} \omega \wedge \omega^{\prime}\right)-d_{\perp}\left(i_{G}(\omega)\right) \wedge \omega^{\prime}=\left[i_{G}, d_{\perp}\right](\omega) \wedge \omega^{\prime} .
$$

(ii) Let $Y_{1}, Y_{2}, X_{1}, \ldots, X_{r} \in \Gamma(\mathscr{F}), Z \in \Gamma(H)$, and $\omega \in \Omega^{r+2,0}(X, \mathscr{F})$ be arbitrary. Using a simple computation, we obtain

$$
\begin{aligned}
& \left(\left[i_{Y_{1} \wedge Y_{2}}, d_{\perp}\right] \omega\right)\left(X_{1}, \ldots, X_{r}, Z\right) \\
& \quad=\omega\left(\pi_{\mathscr{F}}\left[Z, Y_{1}\right], Y_{2}, X_{1}, \ldots, X_{r}\right)-\omega\left(\pi_{\mathscr{F}}\left[Z, Y_{2}\right], Y_{1}, X_{1}, \ldots, X_{r}\right),
\end{aligned}
$$

where $\pi_{\mathscr{F}}$ is the projection onto $\mathscr{F}$ along $H$. Therefore, we get:

$$
\left[i_{Y_{1} \wedge Y_{2}}, d_{\perp}\right]=i_{\pi \mathscr{F}\left[Z, Y_{1}\right] \wedge Y_{2}-\pi \mathscr{F}\left[Z, Y_{2}\right] \wedge Y_{1}} .
$$

By direct inspection from the definition of the Schouten-Nijenhuis bracket, we deduce that

$$
\left[i_{Y_{1} \wedge Y_{2}}, d_{\perp}\right]=i_{\pi_{\Lambda} 2 \mathscr{F}}\left(\left[Y_{1} \wedge Y_{2}, Z\right]_{S N}\right)
$$


where $\pi_{\Lambda^{2} \mathscr{F}}$ is the projection onto the space of longitudinal $(2,0)$-vectors. But since $\omega \in \Omega^{r+2,0}(X, \mathscr{F})$, this completes the proof.

We continue to assume for the rest of this section that $(X, \mathscr{F})$ is a longitudinally symplectic foliation with $\operatorname{dim}(\mathscr{F})=2 p$ and $\operatorname{codim}(\mathscr{F})=q$. For any leaf $L$ of the foliation $\mathscr{F}$ of $X$, let $\omega_{L}$ be the symplectic two form of $L$. Then there exists longitudinal 2-forms on $X$ that restrict on each leaf $L$ to $\omega_{L}$. If we use the splitting (2) then we can choose in a unique way a differential 2-form $\omega \in \Omega^{2,0}(X, \mathscr{F})$ that restricts to $\omega_{L}$ on each leaf $L$. The form $\omega$ will be called the longitudinal symplectic form of $(X, \mathscr{F})$. It depends on the splitting (2).

Using the longitudinal symplectic form $\omega$ we can recover the longitudinal volume form associated with the symplectic orientation by setting:

$$
\operatorname{vol}_{\mathscr{F}}(X):=\frac{1}{p !} \omega^{p} .
$$

We then define the longitudinal symplectic Hodge operator $*_{\mathscr{F}}: \Omega^{r, 0}(X, \mathscr{F}) \rightarrow$ $\Omega^{2 p-r, 0}(X, \mathscr{F})$ by the equality:

$$
\beta \wedge(* \mathscr{F} \alpha)=(\beta, \alpha)_{\omega} \operatorname{vol}_{\mathscr{F}}(X), \quad \forall \alpha, \beta \in \Omega^{r, 0}(X, \mathscr{F}),
$$

where $(\cdot, \cdot)_{\omega}$ is the bilinear form induced by the symplectic form on longitudinal differential forms.

Remark 3.7. For any $f \in \mathscr{C}^{\infty}(X)$ we have by the definition of $* \mathscr{F}$ :

$$
* \mathscr{F}(f \alpha)=f * \mathscr{F} \alpha .
$$

Recall now (Definition 1.2) that $\mathrm{H}^{r, s}(X, \mathscr{F})$ denotes the $r^{\text {th }}$ cohomology group of the longitudinal complex

$$
0 \longrightarrow \Omega^{0, s}(X, \mathscr{F}) \stackrel{d_{\mathscr{F}}}{\longrightarrow} \Omega^{1, s}(X, \mathscr{F}) \stackrel{d_{\mathscr{F}}}{\longrightarrow} \cdots \stackrel{d_{\mathscr{F}}}{\longrightarrow} \Omega^{2 p, s}(X, \mathscr{F}) \longrightarrow 0,
$$

and $\mathrm{H}_{r, S}^{\delta_{\mathscr{F}}}(X, \mathscr{F})$ is the longitudinal Poisson homology of $(X, \mathscr{F})$, that is, the $r^{\text {th }}$-cohomology group of the complex

$$
0 \longrightarrow \Omega^{2 p, s}(X, \mathscr{F}) \stackrel{\delta_{\mathscr{F}}}{\longrightarrow} \Omega^{2 p-1, s}(X, \mathscr{F}) \stackrel{\delta_{\mathscr{F}}}{\longrightarrow} \cdots \stackrel{\delta_{\mathscr{F}}}{\longrightarrow} \Omega^{0, s}(X, \mathscr{F}) \longrightarrow 0 .
$$

The cohomology $\mathrm{H}_{*}^{\delta_{\mathscr{F}}}(X, \mathscr{F})$ of the global complex $\left(\Omega^{k}(X)\right)_{0 \leq k \leq 2 p+q}$ with respect to the operator $\delta_{\mathscr{F}}$ is hence given by

$$
\mathrm{H}_{k}^{\delta_{F}}(X, \mathscr{F}) \simeq \bigoplus_{r+s=k} \mathrm{H}_{r, s}^{\delta_{\mathscr{F}}}(X, \mathscr{F})
$$


We hope the reader will be able to easily tell apart all these cohomology groups and distinguish for instance the groups $\mathrm{H}_{*}^{\delta_{F}}(X, \mathscr{F})$ from the Poisson homology groups of $X$ that are denoted $\mathrm{H}_{*}^{\delta}(X)$, (see the end of the introduction for a list of references to the definitions of the main cohomology groups).

When the foliation $(X, \mathscr{F})$ is longitudinally symplectic, the longitudinal symplectic Hodge operator extends to a well defined operator, still denoted $* \mathscr{F}$,

$$
*_{\mathscr{F}}: \Omega^{r, s}(X, \mathscr{F}) \rightarrow \Omega^{2 p-r, s}(X, \mathscr{F}), \quad \forall s \in\{0, \ldots, q\} .
$$

defined by

$$
*_{\mathscr{F}}(\alpha \wedge \beta):=*_{\mathscr{F}}(\alpha) \wedge \beta,
$$

for any $\alpha \in \Omega^{r, 0}(X, \mathscr{F})$ and any $\beta \in \Omega^{0, s}(X, \mathscr{F})$. This is a consequence of the relation (16) and the splitting (2). We then see that $*_{\mathscr{F}}^{2}=1$.

We point out that the longitudinal Poisson differential $\delta_{\mathscr{F}}$ also satisfies a similar relation, namely

$$
\delta_{\mathscr{F}}(\alpha \wedge \beta):=\delta_{\mathscr{F}}(\alpha) \wedge \beta,
$$

which follows from the formula given for $\delta_{\mathscr{F}}$ in Proposition 3.5 using the same method as in [3].

Proposition 3.8 (Vaisman). Let $(X, \mathscr{F})$ be a longitudinally symplectic foliated manifold with leaves of dimension $2 p$.

(1) We have $(-1)^{r+1} * \mathscr{F} \circ d_{\mathscr{F}} \circ * \mathscr{F}=\delta_{\mathscr{F}}$, on $\Omega^{r, s}(X, \mathscr{F})$.

(2) The cohomology of $X$ with respect to $\delta_{\mathscr{F}}$ is given by

$$
\mathrm{H}_{r, s}^{\delta_{\mathscr{F}}}(X, \mathscr{F}) \cong \mathrm{H}^{2 p-r, s}(X, \mathscr{F}),
$$

and hence $\mathrm{H}_{k}^{\delta_{F}}(X, \mathscr{F}) \cong \oplus_{0 \leq j \leq k} \mathrm{H}^{2 p-j, k-j}(X, \mathscr{F})$.

Proof. The proof of (1) is in [24, page 80]. It can also be derived easily from the properties listed above.

(2) We have $\mathrm{H}_{k}^{\delta_{F}}(X, \mathscr{F}) \simeq \bigoplus_{k=r+s} \mathrm{H}_{r, s}^{\delta_{F}}(X, \mathscr{F})$. The above result (2), shows that $* \mathscr{F}$ induces an isomorphism $\mathrm{H}_{r, s}^{\delta_{F}}(X, \mathscr{F}) \simeq \mathrm{H}^{2 p-r, s}(X, \mathscr{F})$, extending the case $s=0$. This proves that $\mathrm{H}_{k}^{\delta_{F}}(X, \mathscr{F}) \simeq \bigoplus_{j=0}^{k} \mathrm{H}^{2 p-j, k-j}(X, \mathscr{F})$, as claimed. See [24] again.

\section{Conic foliations and their cohomology}

We now introduce the action of $\mathbf{R}_{+}^{*}$ into the picture.

Definition 4.1. Let $(X, \mathscr{F}, G)$ be a longitudinally symplectic foliation. The triple $(X, \mathscr{F}, G)$ will be called a longitudinally symplectic conic foliation 
if there exists a free smooth action of the group $\mathrm{R}_{+}^{*}$ on $X$ by leaf-preserving diffeomorphisms $\left(\alpha_{t}\right)_{t>0}$ such that $\left(\alpha_{t}\right)_{*}(G)=G / t$.

This definition means that each leaf is a conic symplectic manifold in the sense of [5] and that the global action $\alpha$ is smooth. For $l \in \mathrm{Z}$, recall that a differential form $\omega \in \Omega^{k}(X)$ is $l$-homogeneous if

$$
\left(\alpha_{t}\right)^{*}(\omega)=t^{l} \omega, \quad \forall t>0 .
$$

We shall denote as before by $\Omega^{k}(X)_{l}$ the space of $l$-homogeneous differential $k$-forms on $X$. From the definition of a longitudinally symplectic conic foliation, we deduce that the longitudinally symplectic form corresponding to the bivector $G$ belongs to $\Omega^{2}(X)_{1}$.

Since the action of $\mathrm{R}_{+}^{*}$ on the longitudinally symplectic conic foliation $(X, \mathscr{F})$ is free, we can choose the complement $H$ to $F$ in $T X$ to be $\mathrm{R}_{+}^{*}$ invariant. The bi-grading on forms is also $\mathrm{R}_{+}^{*}$-equivariant and we shall denote, as before, by $\Omega^{r, s}(X, \mathscr{F})_{l}$ the smooth $l$-homogeneous sections of $\Lambda^{r} \mathscr{F}^{*} \otimes \Lambda^{s} v^{*}$.

Let now $(M, F)$ be a smooth foliated manifold and take $X=F^{*} \backslash M$. We are interested in the foliated manifold $(X, \mathscr{F})$, where $\mathscr{F}$ is the foliation defined on the total space $F^{*}$ of the longitudinal cotangent bundle to $(M, F)$ as before and then restricted to $X=F^{*} \backslash M$. The radial action of $\mathrm{R}_{+}^{*}$ allows us to consider $l$-homogeneous forms $\Omega^{k}(X)_{l}$ and $\Omega^{r, s}(X, \mathscr{F})_{l}$. As we have already observed, the foliated manifold $(X, \mathscr{F})$ is then longitudinally symplectic. The Poisson differential $\delta$ associated with the Poisson structure of $X$ sends $\Omega^{k}(X)_{l}$ to $\Omega^{k-1}(X)_{l-1}$. The same holds for the operators $\delta_{\mathscr{F}}$ and $\delta_{-2,1}$ defined in the previous section.

Definition 4.2. We denote by $\mathrm{H}_{k}^{\delta}(X)_{l}$ the $l$-homogeneous Poisson homology of $X$, defined by

$$
\mathrm{H}_{k}^{\delta}(X)_{l}:=\frac{\operatorname{Ker}\left(\delta: \Omega^{k}(X)_{l} \rightarrow \Omega^{k-1}(X)_{l-1}\right)}{\delta\left(\Omega^{k+1}(X)_{l+1}\right)} .
$$

In the same way, using again the splitting (2), we set

$$
\mathrm{H}_{r, s}^{\delta_{\mathscr{F}}}(X, \mathscr{F})_{l}:=\frac{\operatorname{Ker}\left(\delta_{\mathscr{F}}: \Omega^{r, s}(X, \mathscr{F})_{l} \rightarrow \Omega^{r-1, s}(X, \mathscr{F})_{l-1}\right)}{\delta_{\mathscr{F}}\left(\Omega^{r+1, s}(X, \mathscr{F})_{l+1}\right)}
$$

and

$$
\mathrm{H}_{k}^{\delta_{\mathscr{F}}}(X, \mathscr{F})_{l}:=\frac{\operatorname{Ker}\left(\delta_{\mathscr{F}}: \Omega^{k}(X)_{l} \rightarrow \Omega^{k-1}(X)_{l-1}\right)}{\delta_{\mathscr{F}}\left(\Omega^{k+1}(X)_{l+1}\right)} .
$$

The homogeneous Poisson complex $\left(\Omega^{*}(X)_{*}, \delta\right)$ splits into a direct sum of 
finite homogeneous subcomplexes $\left(\mathscr{P}^{k}\right)_{k \in Z}$ defined by:

$$
\mathscr{P}^{k}: 0 \longrightarrow \mathscr{P}_{2 p+q-k}^{k} \stackrel{\delta}{\longrightarrow} \mathscr{P}_{2 p+q-k-1}^{k} \stackrel{\delta}{\longrightarrow} \cdots \stackrel{\delta}{\longrightarrow} \mathscr{P}_{-k}^{k} \longrightarrow 0,
$$

where $\mathscr{P}_{l}^{k}:=\Omega^{k+l}(X)_{l}$. Therefore we have:

$$
\mathrm{H}_{k+l}^{\delta}(X)_{l}=\frac{\operatorname{Ker}\left(\delta: \mathscr{P}_{l}^{k} \rightarrow \mathscr{P}_{l-1}^{k}\right)}{\delta\left(\mathscr{P}_{l+1}^{k}\right)} .
$$

If we define in the same way $\mathscr{P}_{l}^{r, s}:=\Omega^{r+l, s}(X, \mathscr{F})_{l}$, then we get a further splitting:

$$
\mathscr{P}_{l}^{k} \simeq \bigoplus_{r+s=k} \mathscr{P}_{l}^{r, s} .
$$

With respect to this splitting, the differential $\delta_{\mathscr{F}}$ preserves $\mathscr{P}^{r, s}:=\oplus_{l \in \mathrm{Z}} \mathscr{P}_{l}^{r, s}$ and sends $\mathscr{P}_{l}^{r, s}$ to $\mathscr{P}_{l-1}^{r, s}$. Thus, to compute the homogeneous homology of the longitudinal Poisson differential $\delta_{\mathscr{F}}$, we can restrict ourselves to $\mathscr{P}^{r, s}$. Note though that the extra differential $\delta_{-2,1}$ does not preserve $\mathscr{P}^{r, s}$ and sends it to $\mathscr{P}^{r-1, s+1}$.

Our next result is that, in order to compute homogeneous Poisson homology, we can get rid of the extra term $\delta_{-2,1}$.

Proposition 4.3. Let $(X, \mathscr{F})$ be a longitudinally symplectic conic foliation. Then

$$
\mathrm{H}_{k+l}^{\delta}(X)_{l} \cong \mathrm{H}_{k+l}^{\delta_{F}}(X, \mathscr{F})_{l} .
$$

Proof. Recall that we have:

$$
\delta=\delta_{\mathscr{F}}+\delta_{-2,1} \quad \text { and } \quad \delta_{\mathscr{F}} \delta_{-2,1}+\delta_{-2,1} \delta_{\mathscr{F}}=0 .
$$

Thus, for any fixed $k$, we use the decomposition $\mathscr{P}^{k} \cong \bigoplus_{i+j=k} \mathscr{P}^{i, j}$ into a finite double complex. We set for any fixed $k \in \mathbf{Z}$,

$$
K^{j, l}:=\mathscr{P}_{l-j}^{k-j, j},
$$

so that

$$
\delta_{\mathscr{F}}: K^{j, l} \longrightarrow K^{j, l-1} \quad \text { and } \quad \delta_{-2,1}: K^{j, l} \longrightarrow K^{j+1, l} .
$$

To compute the homogeneous $\delta$-homology of $X$, we use that the complex splits into the subcomplexes $\left(\mathscr{P}^{k}, \delta\right)$. Therefore, we can fix the integer $k \in \mathbf{Z}$ and define a filtration of the above bicomplex $K^{j, l}$ by setting

$$
F_{h}:=\bigoplus_{l \in Z, j \leq h} K^{j, l} .
$$


This yields a spectral sequence $\left(E^{r}\right)_{r \geq 1}$ which converges to the $\delta$-homology because it comes from a filtration that is bounded both below and above. The $E^{1}$ term of this spectral sequence is computed by a de Rham cohomology group

$$
E_{u, v}^{1} \simeq \mathrm{H}^{2 p-v-k+u, u}(X, \mathscr{F})_{p-k+u},
$$

the isomorphism being implemented by the leafwise symplectic duality operator $* \mathscr{F}$. We now observe that the homogeneous longitudinal de Rham cohomology space $\mathrm{H}^{2 p-v-k+u, u}(X, \mathscr{F})_{p-k+u}$ is trivial unless $u=k-p$, by the homotopy invariance of de Rham cohomology. Therefore, we get

$$
E_{u, v}^{1}=0 \quad \text { if } \quad v \neq-k-p .
$$

Hence for any $r \geq 1$, we see that $d^{r}=0$ and the spectral sequence collapses at $E^{1}$. The proof is thus complete since the spectral sequences considered are convergent.

COROLlary 4.4. Let $(X, \mathscr{F})$ be a longitudinally symplectic conic foliation with leaves of dimension $2 p$. Then $\mathrm{H}_{r, s}^{\delta_{\mathscr{F}}}(X, \mathscr{F})_{l} \cong \mathrm{H}^{2 p-r, s}(X, \mathscr{F})_{l+p-r}$, and hence

$$
\mathrm{H}_{k}^{\delta_{\mathscr{F}}}(X, \mathscr{F})_{l} \cong \bigoplus_{0 \leq j \leq k} \mathrm{H}^{2 p-j, k-j}(X, \mathscr{F})_{l+p-j}
$$

Proof. This is a consequence of Proposition 3.8. Note that we have:

$$
\mathrm{H}_{k}^{\delta_{\mathscr{F}}}(X, \mathscr{F})_{l} \simeq \bigoplus_{r+s=k} \mathrm{H}_{r, s}^{\delta_{\mathscr{F}}}(X, \mathscr{F})_{l} .
$$

But by definition of the operator $* \mathscr{F}$, we see that it sends $l$-homogeneous forms of bi-degree $(r, s)$ to $(l+p-r)$-homogeneous forms of bi-degree $(2 p-r, s)$.

In the case of trivial foliations by 2-planes, that is, when $X=\mathrm{R}^{2} \times \mathrm{R}^{q}$ foliated by the symplectic planes $\mathrm{R}^{2} \times\{p t\}$, if we denote by $(x, \xi)$ the symplectic coordinates along the leaves and $\left(y_{1}, \ldots, y_{q}\right)$ the transverse coordinates, we have the following easy generalizations of some equations in [4]. Namely, for any $f, g, h \in \mathscr{C}^{\infty}(X)$ :

$$
\begin{gathered}
* \mathscr{F}(f)=f d x d \xi, \quad * \mathscr{F}(f d x+g d \xi)=-(f d x+g d \xi), \\
* \mathscr{F}\left(h d y_{i}\right)=h d x d \xi d y_{i} \quad \text { and } \quad * \mathscr{F}(f d x * d \xi)=f .
\end{gathered}
$$

In the same way we have

$$
\begin{aligned}
& * \mathscr{F}\left(f d x d y_{i_{1}} d y_{i_{2}} \ldots d y_{i_{k}}\right)=-\left(f d x d y_{i_{1}} d y_{i_{2}} \ldots d y_{i_{k}}\right), \\
& *_{\mathscr{F}}\left(f d \xi d y_{i_{1}} d y_{i_{2}} \ldots d y_{i_{k}}\right)=-\left(f d \xi d y_{i_{1}} d y_{i_{2}} \ldots d y_{i_{k}}\right),
\end{aligned}
$$




$$
\begin{gathered}
*_{\mathscr{F}}\left(f d y_{i_{1}} d y_{i_{2}} \ldots d y_{i_{k}}\right)=f d x d \xi d y_{i_{1}} d y_{i_{2}} \ldots d y_{i_{k}} \\
\text { and } \quad * \mathscr{F}\left(f d x d \xi d y_{i_{1}} d y_{i_{2}} \ldots d y_{i_{k}}\right)=f d y_{i_{1}} d y_{i_{2}} \ldots d y_{i_{k}} .
\end{gathered}
$$

On the other hand,

$$
\{f, g\}=\frac{\partial f}{\partial \xi} \frac{\partial g}{\partial x}-\frac{\partial f}{\partial x} \frac{\partial g}{\partial \xi} .
$$

Hence Propositions 3.8 and 4.4 can also be proved by reducing to the above trivial case, as in [4], for example.

Remark 4.5. The operator $\delta_{-2,1}$ gives rise to a new differential

$$
d_{2,1}=(-1)^{r+1} * \mathscr{F} \circ \delta_{-2,1} \circ * \mathscr{F}
$$

on $\Omega^{r, s}(X, \mathscr{F})$ whose bi-degree is $(2,1)$ and which satisfies

$$
d_{2,1}^{2}=0, \quad d_{2,1} d_{\mathscr{F}}+d_{\mathscr{F}} d_{2,1}=0 .
$$

We are now in position to compute the homogeneous Poisson homology of a longitudinally symplectic conic foliation. The homogeneous Poisson homology spaces $\mathrm{H}_{k}^{\delta}(X)_{l}$ were defined in Definition 4.2.

THEOREM 4.6. Let $(X, \mathscr{F})$ be a longitudinally symplectic conic foliation. We denote $\operatorname{dim}(\mathscr{F})=2 p$ and $\operatorname{codim}(\mathscr{F})=q$, as before. Then

$$
\mathrm{H}_{k}^{\delta}(X)_{l} \cong \mathrm{H}^{p-l, k-l-p}(X, \mathscr{F})_{0}
$$

for $0 \leq k \leq 2 p+q$ and $|l| \leq p$. For the other values of $k$ and $l$ we have $\mathrm{H}_{k}^{\delta}(X)_{l}=0$.

Proof. We use Proposition 4.3 to conclude that $\mathrm{H}_{k+l}^{\delta}(X)_{l} \simeq \mathrm{H}_{k+l}^{\delta_{F}}(X, \mathscr{F})_{l}$. By Proposition 4.4,

$$
\mathrm{H}_{k}^{\delta_{F}}(X, \mathscr{F})_{l} \cong \oplus_{0 \leq j \leq k} \mathrm{H}^{2 p-j, k-j}(X, \mathscr{F})_{l+p-j} .
$$

By the homotopy invariance of de Rham cohomology, only the groups for which $l+p-j=0$, are non-zero. The result is obtained then by substituting $j=p+l$. The other groups vanish for dimension reasons.

Let now $X=F^{*} \backslash M$ be the dual of the foliation $F$ of $M$ with the zero section removed and with the induced structure of a longitudinal symplectic conic foliation. Let $\mathrm{S}^{*} F=X / \mathrm{R}_{+}^{*}$ be the cosphere bundle of $F$ and $F_{1}$ be the induced foliation on $\mathrm{S}^{*} F \times \mathrm{S}^{1}$ with leaves of dimension $2 p$ (each copy of $\mathrm{S}^{1}$ is completely contained in a leaf). The above theorem then gives the following result. 
Corollary 4.7. Let $(M, F)$ be a foliated manifold and $X=F^{*} \backslash$ $M, \mathrm{~S}^{*} F=X / \mathrm{R}_{+}^{*}$, and $F_{1}$ be as in the paragraph above. We denote $p=$ $\operatorname{dim}\left(F_{1}\right) / 2=\operatorname{dim}(F)$ and $q=\operatorname{codim}\left(F_{1}\right)=\operatorname{codim}(F)$. Then

$$
\begin{aligned}
\mathrm{H}_{k}^{\delta}(X)_{l} & \cong \mathrm{H}^{p-l, k-l-p}\left(\mathbf{S}^{*} F \times \mathbf{S}^{1}, F_{1}\right) \\
& \cong \mathrm{H}^{p-l, k-l-p}\left(\mathbf{S}^{*} F, \mathbf{S}^{*} F \cap F_{1}\right) \oplus \mathrm{H}^{p-l-1, k-l-p}\left(\mathbf{S}^{*} F, \mathbf{S}^{*} F \cap F_{1}\right)
\end{aligned}
$$

for $0 \leq k \leq 2 p+q$ and $|l| \leq p$. For the other values of $k$ and $l$, we have $\mathrm{H}_{k}^{\delta}(X)_{l}=0$.

Proof. The proof is exactly as in the case when the foliation $F$ is trivial (with just one leaf) [2], [3], [5], [14]. The crucial ingredient of the proof is to choose a function $r \neq 0$ homogeneous of degree one. Then identify $r^{-1} d r$ with $\omega_{1}$, the generator of $H^{1}\left(\mathbf{S}^{1}\right)$. This leads to the isomorphism $\mathrm{H}^{p-l, k-l-p}(X, \mathscr{F})_{0}$ $\cong \mathrm{H}^{p-l, k-l-p}\left(\mathbf{S}^{*} F \times \mathbf{S}^{1}, F_{1}\right)$. The second isomorphism follows from Corollary 2.6.

We are ready now to handle an explicit example.

ExAmple 4.8. Let us consider $M=\left(\mathbf{S}^{1}\right)^{n}$, foliated by the one parameter subgroups $\left(e^{2 \pi l \alpha_{1} t}, e^{2 \pi l \alpha_{2} t}, \ldots, e^{2 \pi l \alpha_{n} t}\right)$, not all of $\alpha_{i}$ 's equal to zero. Thus $p=1$ and $q=n-1$. Then

$$
F=M \times \mathrm{R}, \quad X=M \times(\mathrm{R} \backslash\{0\}), \quad \mathrm{S}^{*} F \times \mathrm{S}^{1} \simeq M \times\{ \pm\} \times \mathrm{S}^{1},
$$

with leaves $L \times\{\epsilon\} \times \mathrm{S}^{1}$, where $L \subset M$ is a leaf of $M$ and $\epsilon=+$ or $\epsilon=-$. Then the second isomorphism in Corollary 4.7 gives

$$
\mathrm{H}^{k, h}\left(\mathrm{~S}^{*} F \times \mathrm{S}^{1}, F_{1}\right) \cong\left(\mathrm{H}^{k, h}(M, F) \oplus \mathrm{H}^{k-1, h}(M, F)\right) \otimes \mathrm{C}^{\{ \pm\}} .
$$

$\left(\mathrm{C}^{\{ \pm\}}\right.$is the complex vector space with basis + and - .) To obtain more precise results (which happen to also be finite dimensional spaces), we shall assume now that there exists $C>0$ and $N \in \mathrm{N}$ such that

$$
\left|m_{1} \alpha_{1}+m_{2} \alpha_{2}+\cdots+m_{n} \alpha_{n}\right|^{-1} \leq C\left(\left|m_{1}\right|+\left|m_{2}\right|+\cdots+\left|m_{n}\right|\right)^{N},
$$

for any $m_{1}, \ldots, m_{n} \in \mathbf{Z}$, not all zero. (When $n=2$, this can be achieved by choosing $\alpha_{2} / \alpha_{1}$ to be an irrational algebraic number, for example.)

Trivialize the normal bundle to $F$ using the standard metric on $M$. Let $s\left(Z^{n}\right)$ be the space of rapidly decreasing functions on $Z^{n}$. The Fourier transform then establishes isomorphisms

$$
\Omega^{k, h}(M, F) \cong s\left(\mathrm{Z}^{n}\right) \otimes \Lambda^{h} C^{n-1}, \quad \text { for } \quad k=0,1,
$$


and $\Omega^{k, h}(M, F)=0$ otherwise. Under these isomorphisms, the differential

$$
d_{F}: \Omega^{0, h}(M, F) \rightarrow \Omega^{1, h}(M, F)
$$

becomes multiplication by $m_{1} \alpha_{1}+m_{2} \alpha_{2}+\ldots+m_{n} \alpha_{n}$. The assumption

of Equation (23) then implies $\mathrm{H}^{k, h}(M, F) \cong \Lambda^{h} \mathrm{C}^{n-1}$, for $k=0,1$, and $\mathrm{H}^{k, h}(M, F)=0$ otherwise. Thus $\mathrm{H}^{k, h}(M, F) \cong \Lambda^{k} \mathrm{C} \otimes \Lambda^{h} \mathrm{C}^{n-1}$, for any $k$ and $h$.

Putting all thes calculations together we obtain

$$
\begin{aligned}
\mathrm{H}^{k, h}\left(\mathrm{~S}^{*} F \times \mathrm{S}^{1}, F_{1}\right) & \cong\left(\Lambda^{k} \mathrm{C} \oplus \Lambda^{k-1} \mathrm{C}\right) \otimes \Lambda^{h} \mathrm{C}^{n-1} \otimes \mathrm{C}^{\{ \pm\}} \\
& \cong \Lambda^{k} \mathrm{C}^{2} \otimes \Lambda^{h} \mathrm{C}^{n-1} \otimes \mathrm{C}^{\{ \pm\}}
\end{aligned}
$$

\section{Complete symbols on foliations}

We shall use the results of the previous sections to study the Hochschild homology of the algebra $\mathscr{A}(M, F)$ of complete symbols of longitudinal, classical pseudodifferential operators along the leaves of a foliation $(M, F)$. We begin by defining the algebra $\mathscr{A}(M, F)$. We assume $M$ to be compact for simplicity. Most of the following results and constructions work for $M$ non-compact, but become notationally more complicated. In particular, the main computations of Hochschild homology, Theorems 6.2 and 6.3 remain true by considering compactly supported cohomology groups. In this section $n$ does not denote the dimension of $M$.

If $(M, F)$ is the foliation defined by the fibers of a fibration $M \rightarrow B$, then $\psi^{\infty}(M, F)$ denotes the space of smooth families of pseudodifferential operators along the fibers of $M \rightarrow B$ and we define $\mathscr{A}(M, F):=\psi^{\infty}(M, F) / \psi^{-\infty}$ $(M, F)$.

To construct the algebra $\mathscr{A}(M, F)$ in general, consider a covering $M=$ $\cup U_{\alpha}$ of $M$ with distinguished open subsets. Then

$$
\mathscr{A}(M, F)=\sum \mathscr{A}\left(U_{\alpha},\left.F\right|_{U_{\alpha}}\right)
$$

where the sum is taken in the space $\prod_{L} \psi^{\infty}(L) / \psi^{-\infty}(L)$, with $L$ ranging through all leaves of $(M, F)$. Note that it still makes sense to talk about complete symbols of order (at most) $m$ in $\mathscr{A}(M, F)$, which provides us with a natural filtration $F_{m} \mathscr{A}(M, F)$ of $\mathscr{A}(M, F)$. (In fact, we can define the algebra $\psi^{\infty}(M, F)$ similarly [6], [16], and then $F_{m} \mathscr{A}(M, F)=\psi^{m}(M, F) / \psi^{-\infty}$ $(M, F)$.)

Let $S^{m}\left(F^{*}\right)$ be the space of classical (compactly supported in the base variable) symbols on the vector bundle $F^{*}$. Using standard procedures, one 
can define a quantization map [19]:

$$
q: S^{\infty}\left(F^{*}\right):=\cup_{m \in Z} S^{m}\left(F^{*}\right) \longrightarrow \psi^{\infty}(M, F),
$$

which maps the subspace $S^{m}\left(F^{*}\right)$ of classical symbols of order $m$ to $F_{m} \mathscr{A}(M, F)$ and satisfies $\sigma_{m}(q(a)) \in a+S^{m-1}\left(F^{*}\right)$ if $a \in S^{m}\left(F^{*}\right)$. (In fact one could define a quantization map $q: S^{\infty}\left(F^{*}\right):=\cup_{m \in Z} S^{m}\left(F^{*}\right) \rightarrow$ $\psi^{\infty}(M, F)$ descending to our quantization map, but we shall not need this.) We can construct $q$ using a covering of $M$ by distinguished open sets and a partition of unity. Or one can use the results of [19]. Our quantization map induces a filtration preserving bijection

$$
S^{\infty}\left(F^{*}\right) / S^{-\infty}\left(F^{*}\right) \longrightarrow \mathscr{A}(M, F)
$$

Denote now by $\mathscr{G}$ the holonomy Lie groupoid associated with the foliation $(M, F)$. The algebra $\mathscr{A}(M, F)$ then coincides with the algebra of complete symbols on $\mathscr{G}$ as defined in [19]. Note that $\mathscr{A}(M, F)$ is not a topological algebra but it satisfies the axioms of a topologically filtered algebra, see [2, Proposition 3]. Recall that an algebra $\mathscr{A}$ with a given topology, is a topologically filtered algebra if there exists an increasing multi-filtration $F_{n, l}^{m} \mathscr{A} \subset \mathscr{A}$,

$$
F_{n, l}^{m} \mathscr{A} \subset F_{n^{\prime}, l^{\prime}}^{m^{\prime}} \mathscr{A}, \quad \text { if } n \leq n^{\prime}, \quad l \leq l^{\prime}, \text { and } m \leq m^{\prime},
$$

by closed, complemented subspaces, satisfying the following properties:

(1) $\mathscr{A}=\cup_{n, l, m} F_{n, l}^{m} \mathscr{A}$;

(2) The union $\mathscr{A}_{n}:=\cup_{m, l} F_{n, l}^{m} \mathscr{A}$ is a closed subspace such that

$$
F_{n, l}^{m} \mathscr{A}=\mathscr{A}_{n} \cap\left(\cup_{j} F_{j, l}^{m} \mathscr{A}\right)
$$

(3) Multiplication maps $F_{n, l}^{m} \mathscr{A} \otimes F_{n^{\prime}, l^{\prime}}^{m} \mathscr{A}$ to $F_{n+n^{\prime}, l+l^{\prime}}^{m} \mathscr{A}$;

(4) The maps

$$
F_{n, l}^{m} \mathscr{A} / F_{n-j, l}^{m} \mathscr{A} \otimes F_{n^{\prime}, l^{\prime}}^{m} \mathscr{A} / F_{n^{\prime}-j, l^{\prime}}^{m} \mathscr{A} \longrightarrow F_{n+n^{\prime}, l+l^{\prime}}^{m} \mathscr{A} / F_{n+n^{\prime}-j, l+l^{\prime}}^{m} \mathscr{A}
$$

induced by multiplication are continuous;

(5) The quotient $F_{n, l}^{m} \mathscr{A} / F_{n-j, l}^{m} \mathscr{A}$ is a nuclear Frechet space in the induced topology;

(6) The natural map

$$
F_{n, l}^{m} \mathscr{A} \longrightarrow \lim _{\leftarrow} F_{n, l}^{m} / F_{n-j, l}^{m} \mathscr{A}, \quad j \rightarrow \infty
$$

is a homeomorphism; and 
(7) The topology on $\mathscr{A}$ is the strict inductive limit of the subspaces $F_{n, n}^{n} \mathscr{A}$, as $n \rightarrow \infty$ (recall that $F_{n, n}^{n} \mathscr{A}$ is assumed to be closed in $F_{n+1, n+1}^{n+1} \mathscr{A}$ ).

(The above definition corrects a typo in [2], where $n-1$ was written instead of $n-j$ in condition (4) of the above definition.)

We have

Proposition 5.1. Let $(M, F)$ be a smooth, compact foliated manifold. The algebra $\mathscr{A}(M, F)$ is a topologically filtered algebra such that $F_{n, l}^{m} \mathscr{A}(M, F)=$ $F_{n} \mathscr{A}(M, F):=\psi^{n}(M, F) / \psi^{-\infty}(M, F)$, is, in particular, independent of $l$ and $m$.

Proof. The algebra $\mathscr{A}(M, F)$ coincides with the algebra of complete symbols $\mathscr{A}(\mathscr{G})$ on the holonomy Lie groupoid $\mathscr{G}$ as studied in [2] and [3]. Thus we get the result by applying Proposition 3 in [2].

The Hochschild, cyclic, and periodic cyclic homology of the algebra $\mathscr{A}(M, F)$ must be defined by taking into account the fact that it is a topologically filtered algebra. This is done in [2] and also in [3]. Fix a metric on $F$ and let $P$ be a pseudodifferential operator of order one such that $\sigma_{1}(P) \equiv r$ (modulo lower order symbols), where $r \in \mathscr{C}^{\infty}\left(F^{*}\right)$ is a distance function to the origin as defined in the previous section. The graded algebra $\operatorname{Gr}(\mathscr{A}(M, F))$ associated to $\mathscr{A}(M, F)$ is commutative, more precisely

$$
G r(\mathscr{A}(M, F)) \simeq \mathscr{C}^{\infty}\left(\mathrm{S}^{*} F\right) \otimes \mathrm{C}\left[r, r^{-1}\right]
$$

with grading given by the powers of $r$.

The tensor products appearing in the Hochschild complex are completed projective tensor products such that $F_{k} \mathscr{H}_{n}(\mathscr{A}) / F_{k+1} \mathscr{H}_{n}(\mathscr{A})$ is a direct sum of spaces isomorphic to $C^{\infty}\left(\mathrm{S}^{*} F \times \mathrm{S}^{*} F \times \cdots \times \mathrm{S}^{*} F\right)$ and such that the natural map

$$
F_{k} \mathscr{H}_{n}(\mathscr{A}) \longrightarrow \lim _{\leftarrow} F_{k} \mathscr{H}_{n}(\mathscr{A}) / F_{k-j} \mathscr{H}_{n}(\mathscr{A}), \quad j \rightarrow \infty
$$

is an isomorphism. (This last property together with $\mathscr{H}_{n}(\mathscr{A})=\cup_{k} F_{k} \mathscr{H}_{n}(\mathscr{A})$ give, by definition, the asymptotic completeness of our Hochschild complex, see [2]).

Periodic cyclic homology for algebras of complete symbols associated with almost differentiable groupoids was computed in [2]. These results include the case of the holonomy groupoid considered in the present paper. For the sake of completeness, let us state the explicit result for foliations.

THEOREM 5.2. Let $(M, F)$ be a smooth, compact foliated manifold as before. Then the periodic cyclic homology of the algebra $\mathscr{A}(M, F)$ of complete 
longitudinal symbols on $(M, F)$ is given by:

$$
\operatorname{HP}_{k}(\mathscr{A}(M, F)) \simeq \bigoplus_{j \in Z} \mathrm{H}^{k+2 j}\left(\mathbf{S}^{*} F \times \mathbf{S}^{1}\right), \quad k=0,1 .
$$

In the same way, the periodic cyclic homology of the algebra $\mathscr{A}_{0}(M, F)$ of longitudinal complete symbols of order $\leq 0$ is:

$$
\operatorname{HP}_{k}\left(\mathscr{A}_{0}(M, F)\right) \simeq \bigoplus_{j \in Z} \mathrm{H}^{k+2 j}\left(\mathbf{S}^{*} F\right), \quad k=0,1 .
$$

\section{Homology of complete symbols}

We now return to the study of the Hochschild homology of $\mathscr{A}(M, F)$. Recall that $(M, F)$ is a foliated smooth compact manifold with $\operatorname{dim}(M)=n$ and $\operatorname{dim}(F)=p$. The codimension of the foliation will be denoted by $q$ so $n=$ $p+q$.

The canonical filtration of the Hochschild complex defined above (following [2]) gives rise to a spectral sequence $\mathrm{EH}_{k, h}^{r}$, by general results about filtered complexes. This spectral sequence has the $\mathrm{EH}^{1}$-term given by

$$
\mathrm{EH}_{k, h}^{1}=\mathrm{HH}_{k+h}(\operatorname{Gr}(\mathscr{A}(M, F)))_{k},
$$

by [2, Lemma 1]. The Hochschild homology of $\operatorname{Gr}(\mathscr{A}(M, F))$ is identified using a combination of the Hochschild-Kostand-Rosenberg (HKR) isomorphism and a result of Connes, which is the analog of the HKR-isomorphism for algebras of smooth functions. We denote by $\Omega^{r}\left(F^{*} \backslash M\right)_{s}$ the set of differential $r$-forms on the manifold $F^{*} \backslash M$ which are positively $s$-homogeneous in the radial direction. Then we have:

$$
\mathrm{HH}_{l}(G r(\mathscr{A}))_{d} \simeq \Omega^{l}\left(\mathrm{~S}^{*} F\right) r^{d} \oplus \Omega^{l-1}\left(\mathbf{S}^{*} F\right) r^{d-1} d r,
$$

the isomorphism being obtained via the Hochschild-Kostant-Rosenberg-Connes map

$$
\chi\left(a_{0}, \ldots, a_{l}\right)=\frac{1}{l !} a_{0} d a_{1} \ldots d a_{l} .
$$

Let $X:=F^{*} \backslash M$, as above. It will be convenient to identify

$$
\Omega^{l}\left(\mathrm{~S}^{*} F\right) r^{d} \oplus \Omega^{l-1}\left(\mathrm{~S}^{*} F\right) r^{d-1} d r
$$

with the subspace $\Omega^{l}(X)_{d} \subset \Omega^{l}(X)$ consisting of $d$-homogeneous $l$-forms on the manifold $X=F^{*} \backslash M$. Also, we endow $X$ with the foliation $\mathscr{F} \subset T X$ whose leaves are the cotangent bundles to the leaves of $(M, F)$ with the zero 
section removed, as we did in Sections 1 and 3. More precisely, if $\pi: F^{*} \rightarrow M$ is the projection and $T_{v} F^{*}=\operatorname{ker} \pi_{*}$ is the vertical tangent bundle to the fibration $F^{*} \rightarrow M$, then $\mathscr{F}$ is the restriction to $X$ of the bundle $\pi^{*}(F)+T_{v} F^{*}$.

Recall that $X=F^{*} \backslash M$ admits a Poisson structure induced by the natural symplectic structure of the leaves of $\mathscr{F}$. Moreover $(X, \mathscr{F})$ is then a conic symplectic foliation in the sense of Definition 4.1. We introduced in Section 3, a Poisson differential $\delta=\delta_{\mathscr{F}}+\delta_{-2,1}: \Omega^{l}(X) \rightarrow \Omega^{l-1}(X)$, such that $\delta\left(\Omega^{l}(X)_{k}\right) \subset \Omega^{l-1}(X)_{k-1}$. We denote as in Section 3 by

$$
\mathrm{H}_{l}^{\delta}(X)_{d}=\frac{\operatorname{ker}\left(\delta: \Omega^{l}(X)_{d} \rightarrow \Omega^{l-1}(X)_{d-1}\right)}{\delta\left(\Omega^{l+1}(X)_{d+1}\right)}
$$

the homogeneous Poisson homology groups of $X=F^{*} \backslash M$.

Proposition 6.1. Let $\chi: \mathrm{HH}_{l}(\operatorname{Gr}(A))_{d} \rightarrow \Omega^{l}\left(F^{*} \backslash M\right)_{d}$ be the HKR isomorphism, and let $d_{1}: E_{k, h}^{1} \rightarrow E_{k-1, h}^{1}$ be the first differential of the spectral sequence associated to $\mathscr{A}(M, F)$ as in [2]. Then

$$
\chi \circ d_{1} \circ \chi^{-1}=-\sqrt{-1} \delta,
$$

and hence $\mathrm{EH}_{k, h}^{2} \simeq \mathrm{H}_{k+h}^{\delta}\left(F^{*} \backslash M\right)_{k}$.

Proof. We apply Theorem 3.1.1 in [4, page 107]. More precisely, if $\sigma \in$ $\mathscr{A}_{m} / \mathscr{A}_{m-1}, \sigma^{\prime} \in \mathscr{A}_{q} / \mathscr{A}_{q-1}$ then there exist

$$
P \in \psi^{m}(M, F) \quad \text { and } \quad P^{\prime} \in \psi^{q}(M, F)
$$

such that $[P]=\sigma$ and $\left[P^{\prime}\right]=\sigma^{\prime}$ (with obvious notations). One needs the expansion of $P \circ P^{\prime}-P^{\prime} \circ P$ into homogeneous terms. Since the quatization map can be chosen with values operators with support small enough, the symbol expansion of the commutator is obtained in the same way as in the classical case, see [9] and [6], [7] for the corresponding results for foliations.

Let $F_{1}=\pi^{*}(F)+T_{v}\left(\mathrm{~S}^{*} F\right) \subset T\left(\mathrm{~S}^{*} F\right)$ be the integrable sub-bundle defined using $F$, as above, but for the cosphere bundle. (By abuse of notation, we shall sometimes denote $F_{1}$ also the integrable sub-bundles defined similarly by $F$ on the fibrations $F^{*} \rightarrow M$, on $F^{*} \backslash M \rightarrow M$, on $\mathrm{S}^{*} F \rightarrow M$, or on $\mathrm{S}^{*} F \times \mathrm{S}^{1} \rightarrow M$. So on $F^{*} \backslash M$ for instance $F_{1}$ coincides with the foliation $\mathscr{F}$ defined in the previous sections.) Now we gather the results of the previous sections and deduce the following theorem.

THEOREM 6.2. Let $(M, F)$ be a smooth, compact foliated manifold and denote by $p$ the dimension of the leaves of $(M, F)$. Let $\left(\mathrm{EH}^{r}, d^{r}\right)_{r \geq 1}$ be the spectral sequence associated to the canonical filtration of the Hochschild complex of the algebra $\mathscr{A}(M, F):=\psi^{\infty}(M, F) / \psi^{-\infty}(M, F)$, then this spectral 
sequence converges to the Hochschild homology of $\mathscr{A}(M, F)$ and its $E^{2}$ term is given by

$$
\mathrm{EH}_{k, h}^{2} \simeq \mathrm{H}^{p-k, h-p}\left(\mathrm{~S}^{*} F \times \mathrm{S}^{1}, F_{1}\right)
$$

Proof. First we have an isomorphism $\mathrm{EH}_{k, h}^{2} \cong \mathrm{H}_{k+h}^{\delta}\left(F^{*} \backslash M\right)_{k}$ given by Proposition 6.1. By Theorem 4.6 we have

$$
\mathrm{H}_{k+h}^{\delta}\left(F^{*} \backslash M, F_{1}\right)_{k} \cong \mathrm{H}^{p-k, h-p}\left(F^{*} \backslash M, F_{1}\right)_{0} .
$$

But this last group coincides with $\mathrm{H}^{p-k, h-p}\left(\mathrm{~S}^{*} F \times \mathrm{S}^{1}, F_{1}\right)$, as we have already checked in the proof of Corollary 4.7.

The convergence of the spectral sequence is then a consequence of $[2$, Lemma 3] by taking $a=2$ in that lemma.

THEOREM 6.3. Let $(M, F)$ be a smooth, compact foliated manifold with $\operatorname{dim}(F)=p$. Then the space $\mathrm{HH}_{0}(\mathscr{A}(M, F))$ is given by:

$$
\mathrm{HH}_{0}(\mathscr{A}(M, F)) \simeq \mathrm{H}^{2 p, 0}\left(\mathrm{~S}^{*} F \times \mathrm{S}^{1}, F_{1}\right) .
$$

Moreover, when $p \geq 2$, we have

$$
\mathrm{HH}_{0}(\mathscr{A}(M, F)) \simeq \mathrm{H}^{p, 0}(M, F) .
$$

Proof. Using the previous results, we need to show that the differentials $d^{r}$ coming into and out of $\mathrm{EH}_{k, h}^{r}$ are trivial if $k+h=0$. But the $E_{k, h}^{2}$ term vanishes unless $-p \leq k \leq p$ and $p \leq h \leq p+q$ where $q$ is the codimension of the foliation. Thus the only term $E_{-k, k}^{2}$ that may be different from 0 is $E_{-p, p}^{2}$. All differentials coming into and out of $E_{-p, p}^{2}$ are seen to vanish because of the geometry of this spectral sequence. More precisely, recall on that $d^{2}$ : $\mathrm{EH}_{k, h}^{2} \rightarrow \mathrm{EH}_{k-2, h+1}^{2}$ while $\mathrm{EH}_{k, h}^{2}$ is only non trivial when $-p \leq k \leq p$ and $p \leq h \leq p+q$. Thus

$$
\left.d^{2}\right|_{\mathrm{EH}_{-p, p}^{2}} \text { is trivial, }
$$

and the range of $d^{2}$ does not intersect $\mathrm{EH}_{-p, p}^{2}$. In the same way,

$$
d^{r}: \mathrm{EH}_{k, h}^{r} \rightarrow \mathrm{EH}_{k-r, h+r-1}^{r}
$$

thus it is trivial when $k=-p$ and $h=p$, and its range is never of the form $\mathrm{EH}_{-p, p}^{r}$. A recursive argument then finishes the proof. By Corollary 2.5(ii), when $2 p-1 \geq p+1$, we have

$$
\mathrm{H}^{2 p, 0}\left(\mathrm{~S}^{*} F \times \mathrm{S}^{1}, F_{1}\right) \simeq \mathrm{H}^{p, 0}(M, F) .
$$

Thus the proof is complete. 
REMARK 6.4. The above restriction $p \geq 2$ corresponds to the connectedness of the total manifold of the bundle $S^{*} F$ and is similar to the restriction on the uniqueness of the Wodzicki trace in the non foliated situation.

REMARK 6.5. A similar argument enables to obtain that

$$
\mathrm{HH}_{2 p+q}(\mathscr{A}(M, F)) \simeq \mathrm{H}^{0, q}(M, F) .
$$

This follows from the Gysin exact sequence, Theorem 2.4.

It was proved in [3] that the above spectral sequence collapses at $E^{2}$ when the given foliation is a smooth fibration. It would be interesting to establish this result in general, because of the following corollary.

COROLlary 6.6. Let $(M, F)$ be a smooth, compact foliated manifold with $\operatorname{dim}(F)=p$. Assume that the spectral sequence associated with Hochschild homology collapses at $E^{2}$, then the Hochschild homology of the algebra of complete longitudinal pseudodifferential symbols on $(M, F)$ is given by:

$$
\mathrm{HH}_{k}(\mathscr{A}(M, F)) \simeq \bigoplus_{j=0}^{q} \mathrm{H}^{2 p+j-k, j}\left(\mathrm{~S}^{*} F \times \mathrm{S}^{1}, F_{1}\right)
$$

Proof. We have

$$
\begin{aligned}
& \mathrm{HH}_{k}(\mathscr{A}(M, F)) \simeq \oplus_{l} \mathrm{EH}_{k-l, l}^{2} \simeq \oplus_{l} \mathrm{H}^{p-k+l, l-p}\left(F^{*} \backslash M, F_{1}\right)_{0} \\
& \simeq \oplus_{j=0}^{q} \mathrm{H}^{2 p+j-k, j}\left(F^{*} \backslash M, F_{1}\right)_{0} .
\end{aligned}
$$

Using Corollary 4.7, it remains to show the convergence of the above spectral sequence, but this was checked in Theorem 6.2.

Let us now formulate the corresponding results for Hochschild cohomology. First, to define Hochschild cohomology, we just dualize the constructions (inductive and projective limits, but keeping the projective limits first) used to define the Hochschild homology complex in [2]. In particular, all cocycles $\phi$ in the definition of Hochschild complex are such that $\phi\left(a_{0}, \ldots, a_{k}\right)=0$ if the sum of the orders of $a_{0}, a_{1}, \ldots, a_{k}$ is less than some fixed number $N$, that is fixed for each $\phi$. The same theorems on the convergence of the associated spectral sequences then hold for Hochschild cohomology (with the same proof).

THEOREM 6.7. Let $(M, F)$ be a smooth, compact foliated manifold with $\operatorname{dim}(F)=p$. Let $F_{1}$ be the foliation of $\mathrm{S}^{*} F \times \mathrm{S}^{1}$ induced by $F$ and of the same codimension as $F$, as above. Then the spectral sequence $\mathrm{EH}_{r}^{k, h}$ associated with the Hochschild cohomology of $\mathscr{A}(M, F)$ converges to Hochschild cohomology and has the $\mathrm{EH}_{2}$-term given by $\mathrm{EH}_{2}^{k, h} \simeq \mathrm{H}_{p-k, h-p}\left(\mathrm{~S}^{*} F \times \mathrm{S}^{1}, F_{1}\right)$. 
In particular, $\mathrm{HH}^{0}(\mathscr{A}(M, F)) \simeq \mathrm{H}_{2 p, 0}\left(\mathrm{~S}^{*} F \times \mathrm{S}^{1}, F_{1}\right)$.

Thus traces are constructed out of $(2 p, 0)$-invariant currents on $\left(\mathrm{S}^{*} F \times\right.$ $\left.\mathrm{S}^{1}, F_{1}\right)$. But for $p \geq 2$, we have a homological identification, similar to the cohomological one obtained in Theorem 2.4:

$$
\mathrm{H}_{2 p, 0}\left(\mathrm{~S}^{*} F \times \mathrm{S}^{1}, F_{1}\right) \simeq \mathrm{H}_{p, 0}(M, F) .
$$

The space $\mathrm{H}_{p, 0}(M, F)$ is the space of closed holonomy invariant p-currents, see [1].

EXAMPLE 6.8. Let us take a closer look at the foliation of the Example 4.8. By duality, we obtain

$$
\mathrm{H}_{k, h}\left(\mathrm{~S}^{*} F \times \mathrm{S}^{1}, F_{1}\right) \cong \Lambda^{k} \mathrm{C}^{2} \otimes \Lambda^{h} \mathrm{C}^{n-1} \otimes \mathrm{C}^{\{ \pm\}} .
$$

This gives us a canonical basis, $\tau_{+}$and $\tau_{-}$, for $\operatorname{HH}^{0}(\mathscr{A}(M, F))$. It also gives that the dimension of $\mathrm{HH}^{l}(\mathscr{A}(M, F))$ is at most the dimension of the space $\Lambda^{l}\left(\mathrm{C}^{n+1}\right) \otimes \mathrm{C}^{2}$, and that these dimensions are equal if, and only if, the spectral sequence $\mathrm{EH}_{r}$ collapses at $\mathrm{EH}_{2}$.

Chose a subtorus of codimension 1 in $M$ that is transverse to the foliation. This gives rise to $n-1$ one-parameter groups of automorphisms of $\mathscr{A}(M, F)$, and hence to $n-1$-derivations $\delta_{1}, \ldots, \delta_{n-1}$ of this algebra. Let $\delta$ be the derivation given by translation along the leaves of the foliation and $\delta_{r}(T)=[\log Q, T]$, where $Q$ is a positive operator of order 1 with principal symbol $r$, as in [14]. Then each of these derivations acts on the Hochschild complex of $\mathscr{A}(M, F)$.

If $D$ is a derivation and $\phi$ is a $l$-cocycle on $\mathscr{A}(M, F)$, then

$$
\left(i_{D} \phi\right)\left(a_{0}, a_{1}, \ldots, a_{l+1}\right):=\phi\left(a_{0} D\left(a_{1}\right), a_{2}, \ldots, a_{l+1}\right)
$$

will be a $(l+1)$-cocycle on $\mathscr{A}(M, F)$. We have $i_{D} i_{D^{\prime}}=-i_{D^{\prime}} i_{D}$, for all $D, D^{\prime} \in\left\{\delta_{1}, \ldots, \delta_{n-1}, \delta, \delta_{r}\right\}$, because all these derivations commute.

A counting argument gives then that there are as many $l$-cocycles of the form $i_{D_{1}} \ldots i_{D_{l}} \tau_{ \pm}$, with $D_{1}, \ldots, D_{l}$ distinct elements in the set $\left\{\delta_{1}, \ldots, \delta_{n-1}, \delta, \delta_{r}\right\}$ as the maximum possible dimension of $\mathrm{HH}^{l}(\mathscr{A}(M, F))$ established above (that is, the dimension of $\Lambda^{l} \mathrm{C}^{n+1} \otimes \mathrm{C}^{2}$.

The algebra $\mathscr{A}(M, F)$ splits canonically as a direct sum

$$
\mathscr{A}(M, F) \cong \mathscr{A}(M, F)_{+} \oplus \mathscr{A}(M, F)_{-}
$$

because the contangent sphere bundle $\mathrm{S}^{*} F$ is disconnected. We next use the inclusion $\mathscr{C}^{\infty}(M) \subset \mathscr{A}(M, F)$ and the fact that $i_{D_{r}}$ induces a morphism of the Hochschild complexes to prove that all the cocycles in Equation (29) are 
distinct. This shows that the spectral sequence $\mathrm{EH}_{r}$ degenerates at $\mathrm{EH}_{2}$. It also gives an explicit determination of a basis of the groups $\mathrm{HH}^{l}(\mathscr{A}(M, F))$ for this foliation. In particular

$$
\mathrm{HH}^{l}(\mathscr{A}(M, F)) \cong \Lambda^{l} \mathrm{C}^{n+1} \otimes \mathrm{C}^{\{ \pm\}} .
$$

\section{REFERENCES}

1. Abouqateb, A., and El Kacimi Alaoui, A., Fonctionnelles invariantes et courants basiques, Studia Math. 143 (2000), 199-219.

2. Benameur, M-T., and Nistor, V., Homology of Complete Symbols and Non-commutative Geometry, "Collected papers on Quantization of singular symplectic quotients", Progr. Math. (2001).

3. Benameur, M-T., and Nistor, V., Homology of algebras of families of pseudodifferential operators, Max Planck preprint 2001.

4. Brylinski, J.-L., A differential complex for Poisson manifolds, J. Differential Geom. 28 (1988), 93-114.

5. Brylinski, J.-L., and Getzler, E., The homology of algebras of pseudo-differential symbols and the non-commutative residue, K-Theory 1 (1987), 385-403.

6. Connes, A., Sur la théorie non commutative de l'integration, Algèbres d'opérateurs, Lecture Notes in Math. 725 (1979), 19-143.

7. Connes, A., A survey of foliations and operator algebras, Proc. Sympos. Pure Math. 38 (1982), 521-628.

8. Connes, A., Non-commutative Geometry, Academic Press, 1994.

9. Gilkey, P. B., Invariance theory, the heat equation and the Atiyah-Singer index theorem, Math. Lect. Series 11, 1984.

10. Guillemin, V., and Sternberg, S., Geometric Asymptotics, Math. Surveys Monogr. 14 (1977).

11. Kassel, C., Le résidu non commutatif (d'apreès M. Wodzicki) (French), Séminaire Bourbaki, Vol. 1988/89 Astérisque 177-178 (1989), Exp. 798, 199-229.

12. Lauter, R., and Moroianu, S., Homology of pseudo-differential operators on manifolds with fibered boundaries, Mainz University preprint 2000, to appear in J. Reine Angew. Math.

13. Lauter, R., and Moroianu, S., Homology of pseudodifferential operators on manifolds with fibered cusps, Preprint 2002.

14. Melrose, R., and Nistor, V., Homology of pseudodifferential operators I. Manifolds with boundary, to appear in Amer. J. Math.

15. Milnor, J., and Stasheff, D., Characteristic Classes, Ann. of Math. Stud. 76 (1974).

16. Moore, C. C., and Schochet, C., Global Analysis on Foliated Spaces, Springer-Verlag 1988.

17. Nest, R., and Tsygan, B., Algebraic index theorem, Comm. Math. Phys. 172 (1995), 223-262.

18. Nest, R., and Tsygan, B., Algebraic index theorem for families, Adv. Math. 113 (1995), 151-205.

19. Nistor, V., and Weinstein, A., and Ping Xu, Pseudodifferential operators on groupoids, Penn State Preprint No. 202 (1997).

20. Plante, J-F., Foliations with measure preserving holonomy, Ann. of Math. 102 (1975), $327-$ 361.

21. Piazza, P., On the index of elliptic operators on manifolds with boundary, J. Funct. Anal. 117 (1993), 308-359.

22. Roger, C., Cohomologie $(p, q)$ des feuilletages et applications. Transversal structure of foliations, (Toulouse, 1982). Astrisque No. 116 (1984), 195-213. 
23. Tondeur, P., Geometry of Foliations, Monogr. Math. (1997).

24. Vaisman, I., Lectures on the Geometry of Poisson Manifolds, Progr. Math., 1994.

25. Wodzicki, M., Excision in cyclic homology and in rational algebraic K-theory, Ann. of Math. 129 (1989), 591-640.

26. Wodzicki, M., an unpublished handwritten preprint dated 1989.

INSTITUT G. DESARGUES

UNIVERSITÉ LYON 1

LYON

FRANCE

E-mail: benameur@igd.univ-lyon1.fr
PENNSYLVANIA STATE UNIVERSITY UNIVERSITY PARK, PA 16802

USA

E-mail: nistor@math.psu.edu 